\title{
GENERALIZED NEWTON METHODS FOR CRACK PROBLEMS WITH NON-PENETRATION CONDITION
}

\author{
M. HINTERMÜLLER* ${ }^{*}$ V.A. KOVTUNENKO ${ }^{\dagger}$, AND K. KUNISCH ${ }^{\diamond}$
}

\begin{abstract}
A class of semismooth Newton methods for unilaterally constrained variational problems modelling cracks under a non-penetration condition are introduced and investigated. On the continuous level, a penalization technique is applied which allows to argue generalized differentiability of the nonlinear mapping associated to its first order optimality characterization. It is shown that the corresponding semismooth Newton method converges locally superlinearly. For the discrete version of the problem, fast local as well as global and monotonous convergence of a discrete semismooth Newton method are proved. A comprehensive report on numerical tests for the two-dimensional Lamé problem with three collinear cracks under the non-penetration condition ends the paper.
\end{abstract}

\section{INTRODUCTION}

Crack problems are important applications in fracture mechanics. In the past, analytical approaches to crack problems were based on linear boundary value problem formulations on non Lipschitzian domains. The aim was to study solution singularities caused by the non-smooth boundary of the domain and to use this information to construct specific singular solutions; see, e.g., $[3,15]$.

Recently, variational methods were developed for the investigation of (unilaterally) constrained crack problems. The inequality constraint imposed on the jump of the displacement at opposite crack faces prevents the nonphysical, thus inconsistent, behavior of overlapping faces which may occur for linear models. An account for the variational technique can be found, e.g., in $[10,11]$. The problem formulation is in terms of a variational inequality involving elements on a Hilbert lattice. Also the variational formulation

1991 Mathematics Subject Classification. 49J40, 49M29, 90C33, 73M25.

Key words and phrases. variational inequality, constrained optimization, complementarity system, semismooth Newton method, active sets, cracks with non-penetration, linear elasticity.

* Department of Computational and Applied Mathematics - CAAM, Rice University, Houston, Texas, USA, and Department of Mathematics, University of Graz, Graz, Austria.

$\dagger$ Department of Mathematics, University of Graz, Graz, Austria, and Lavrent'ev Institute of Hydrodynamics, Novosibirsk, Russia.

${ }^{\diamond}$ Department of Mathematics, University of Graz, Graz, Austria. 
provides the appropriate state space for the solutions to crack problems which are singular at the crack tip.

Finite element and boundary element methods were proposed for the numerical solution of linear crack problems [7, 14]. While the discretization technique accounting for solution singularity is still an important ingredient, for unilaterally constrained crack problems the numerical solution algorithms are considerably more involved. In $[5,6,13]$ penalty and activeset-type algorithms were proposed and applied for symmetric as well as non-symmetric crack problems. In the corresponding test runs it turned out that the active-set-type approach is very efficient with respect to both the number of iterations required for solving the discretized problems and the amount of work needed per iteration. The first aspect is related to the interpretation of the active-set method as a particular realization of a semismooth Newton method. The second fact is due to the active-set nature of the algorithm which allows to solve, in every iteration, only a linear system on the actual estimate for the inactive set at the solution. The inactive set represents the non-touching part of the crack surfaces.

In the present paper, our aim is to provide a local as well as global convergence analysis for the solution of the continuous and the discrete constrained crack problems by semismooth Newton methods. In general terms, the class of problems considered in this paper is related to the minimization of a convex quadratic objective functional subject to a linear inequality constraint: (1) minimize $\frac{1}{2}\langle L u, u\rangle_{H^{\star}, H}-\langle f, u\rangle_{H^{\star}, H}$ over $u \in H$ subject to $\Lambda u \geq 0$,

where $H$ is a Hilbert space and $H^{\star}$ denotes its dual, $L: H \rightarrow H^{\star}$ is a strongly monotone bounded linear operator, $f \in H^{\star}$ is given, and the linear continuous operator $\Lambda$ maps $H$ onto some Hilbert space $H_{B}$. Let $H_{B}^{\star}$ denote the dual space of $H_{B}$. For crack problems subject to the non-penetration condition the operator $\Lambda$ is related to the jump of traces of the function $u$ across a crack. The unique solution $u$ of (1) can be characterized by the existence of a multiplier $\lambda \in H_{B}^{\star}$ satisfying

$$
\langle L u, v\rangle_{H^{\star}, H}-\langle\lambda, \Lambda v\rangle_{H_{B}^{\star}, H_{B}}-\langle f, v\rangle_{H^{\star}, H}=0 \quad \text { for all } v \in H,
$$

Utilizing a duality operator $J: H_{B} \rightarrow H_{B}^{\star}$ the inequality (2b) can be rewritten as the non-linear equation

$$
0=\Lambda u-P\left(\Lambda u-\alpha J^{-1} \lambda\right):=\Phi(u, \lambda)
$$

for any constant $\alpha>0$, where $P$ denotes the projection onto the nonnegative elements in $H_{B}$. To obtain a more amenable representation of $(2 \mathrm{~b})$, the Lagrange multiplier $\lambda \in H_{B}^{\star}$ has to enjoy certain regularity properties. For general crack problems, however, we only have $\lambda \in H_{B}^{\star}$ which does not allow to, e.g., replace (3) by a representation in the pointwise almost everywhere sense. 
On the function space level this fact prevents the straightforward application of generalized differentiability notions (see $[1,4]$ ) to the projection operator involved in (3). However, utilizing a penalization technique, in section 2 we obtain an approximate multiplier which enjoys extra regularity properties. To some extent, this technique allows to choose $J=\mathrm{id}_{H_{B}}$ while simultaneously relaxing the equality (3). Then $P$ becomes the max-operator and a pointwise almost everywhere interpretation is valid. As a consequence, relying on the concept of semismoothness of a not necessarily (Fréchet) differentiable operator $F$ between Banach spaces, we are able to prove a locally superlinear convergence rate of a semismooth Newton technique associated to the penalized problem for fixed penality parameters. The notion of semismoothness of a real-valued function was introduced in [16], extended to $\mathbb{R}^{n}$ in [18], and carried over to function spaces in [1, 4, 19]. In [4] the following definition is used: The operator $F: X \rightarrow Y$, with $X, Y$ Banach spaces, is slantly differentiable in an open subset $U \subset X$ if there exists a family of mappings $G: U \rightarrow \mathcal{L}(X, Y)$, referred to as generalized derivative, such that

$$
\lim _{h \rightarrow 0} \frac{1}{\|h\|_{X}}\|F(y+h)-F(y)-G(y+h) h\|_{Y}=0
$$

for every $y \in U$. In the present paper we rely on this characterization since it resembles the semismoothness concept in [18] and is valid in function spaces as well as in $\mathbb{R}^{n}$.

In contrast to the situation in the function space setting, after discretization in finite dimensions no problems with respect to generalized differentiability are encountered. Therefore, there is no need for resorting to a penalization technique, rather we introduce the semismooth Newton method for a linear complementarity problem reformulation of the discrete constrained crack problem and analyze its convergence. In fact, we can show fast local as well as monotonous global convergence of the method.

In section 2 we start by introducing the precise functional analytic formulation of the unilaterally constrained crack problem. Further we apply a penalization technique, derive its first order optimality characterization which, is the basis for defining a semi-smooth Newton-type algorithm for the numerical solution. The section ends with a convergence analysis of the Newton process. Section 3 concentrates on the discretized crack problem. We introduce the corresponding discrete semismooth Newton method and analyse its local convergence. The second part of section 3 focusses on the monotonous global convergence of the method. Section 4 is devoted to numerics. We provide results obtained by the discretized penalization technique as well as the semismooth Newton method of section 3. The problem under investigation is a two-dimensional Lamé problem with three collinear cracks. 


\section{The Semismooth NeWton method for CONTINuOus PRoblems}

2.1. Unilaterally constrained crack problems. Let $\Omega \subset \mathbb{R}^{d}$ for $d=2,3$ be a bounded domain with Lipschitz boundary $\partial \Omega$ consisting of two disjoint sets, a Neumann boundary $\Gamma_{N}$ and a Dirichlet boundary $\Gamma_{D} \neq \emptyset$. We assume that inside the domain $\Omega$ we have a crack $\Gamma_{C}$, which is given by some sufficiently smooth surface for $d=3$ or curve for $d=2$. For the theoretical part of the presentation we suppose that $\Gamma_{C} \cap \partial \Omega=\emptyset$ and that the crack does not intersect itself. Let $\nu=\left(\nu_{1}, \ldots, \nu_{d}\right)^{\top}$ denote the unit normal vector at the crack, and corresponding to $\Gamma_{C}$ we define its positiveoriented face $\Gamma_{C}^{+}$and its negative-oriented face $\Gamma_{C}^{-}$, which are obtain as the limit of sequences of points $x=\left(x_{1}, \ldots, x_{d}\right)^{\top} \in \mathbb{R}^{d}$ converging from opposite sides to $\Gamma_{C}$, respectively. We further define $\Omega_{C}=\Omega \backslash \bar{\Gamma}_{C}$ to be the domain $\Omega$ with the crack $\Gamma_{C}$. Its boundary $\partial \Omega_{C}$ consists of $\partial \Omega, \Gamma_{C}^{+}, \Gamma_{C}^{-}$, and is not Lipschitz due to the presence of the crack. See Figure 1 for a graphical account for the geometrical situation described above.

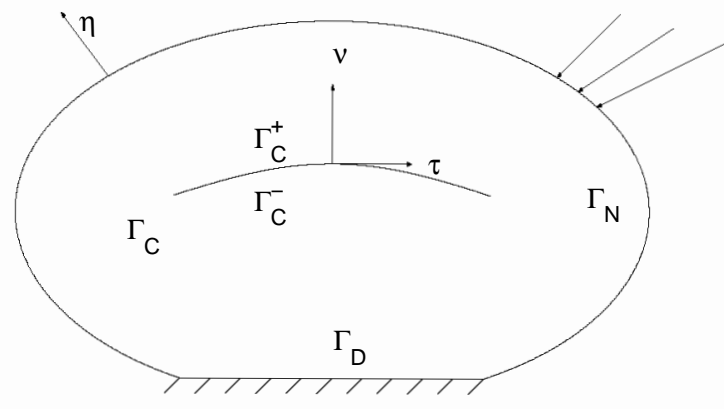

Figure 1. Domain $\Omega_{C}$ with the crack $\Gamma_{C}$.

We suppose that the underlying physics are well described by the linear elasticity model of a solid occupying the domain $\Omega_{C}$. It involves the displacement vector $u=\left(u_{1}, \ldots, u_{d}\right)^{\top}(x)$ in terms of linear, symmetric stress and strain tensors

$$
\sigma_{i j}(u)=c_{i j k l}(x) \varepsilon_{k l}(u), \quad \text { and } \quad \varepsilon_{i j}(u)=0.5\left(u_{i, j}+u_{j, i}\right), \quad i, j=1, \ldots, d,
$$

respectively, with a bounded and symmetric tensor $c$, i.e.,

$$
c_{i j k l}=c_{j i k l}=c_{k l i j}, \quad c_{1} \xi_{i j} \xi_{i j} \leq c_{i j k l} \xi_{k l} \xi_{i j} \leq c_{2} \xi_{i j} \xi_{i j} \quad \text { for all } \xi_{i j}=\xi_{j i}
$$

for some constants $0<c_{1} \leq c_{2}$. Here and throughout we utilize the standard tensor notation common in solid mechanics and the summation convention for repeated indices.

The displacement vector $u$ is considered in the Hilbert space

$$
H\left(\Omega_{C}\right)=\left\{u \in H^{1}\left(\Omega_{C}\right)^{d}: \quad u=0 \quad \text { on } \Gamma_{D}\right\},
$$


and its traces $u^{ \pm}=\left.u\right|_{\Gamma_{C}^{ \pm}}$are defined on the crack faces $\Gamma_{C}^{ \pm}$in the usual way as elements of the space $H^{1 / 2}\left(\Gamma_{C}\right)^{d}$. The jump of the displacement across the crack $\Gamma_{C}$ is denoted by $\llbracket u \rrbracket:=u^{+}-u^{-}$. In [11] (see also [10]) it was observed that physical consistency of the crack model is related to a nonpenetration condition avoiding overlapping crack faces. In mathematical terms this is described by the requirement $u \in K$ with

$$
K=\left\{u \in H\left(\Omega_{C}\right): \quad \llbracket u_{i} \rrbracket \nu_{i} \geq 0 \quad \text { on } \Gamma_{C}\right\} .
$$

Note that $K$ is a closed, convex subset of $H\left(\Omega_{C}\right)$. We refer to $K$ as the set of admissible displacements. For a given surface traction $g=\left(g_{1}, \ldots, g_{d}\right)^{\top} \in$ $L^{2}\left(\Gamma_{N}\right)^{d}$ the displacement in our crack model is the solution to

$$
\text { minimize } \frac{1}{2} \int_{\Omega_{C}} \sigma_{i j}(u) \varepsilon_{i j}(u) d x-\int_{\Gamma_{N}} g_{i} u_{i} d s \quad \text { subject to } u \in K .
$$

The corresponding first order necessary and sufficient optimality condition is given by the variational inequality problem:

Find $u \in K$ such that

$$
\int_{\Omega_{C}} \sigma_{i j}(u) \varepsilon_{i j}(v-u) d x \geq \int_{\Gamma_{N}} g_{i}(v-u)_{i} d s \quad \text { for all } v \in K .
$$

Using Korn's inequality it is standard to argue the existence of a unique solution $u \in K$ to $(5)$, respectively $(P)$.

It can be verified that $\llbracket u \rrbracket \in H_{00}^{1 / 2}\left(\Gamma_{C}\right)^{d}$, where $H_{00}^{1 / 2}\left(\Gamma_{C}\right)$ is the space of functions in $H^{1 / 2}\left(\Gamma_{C}\right)$ which admit a continuation by zero on an extension $\tilde{\Gamma}_{C}$ of $\Gamma_{C}$ into $\Omega_{C}$. Since the trace of $H\left(\Omega_{C}\right)$ onto $H_{00}^{1 / 2}\left(\Gamma_{C}\right)^{d}$ is surjective there exists a Lagrange multiplier $\lambda \in H_{00}^{1 / 2}\left(\Gamma_{C}\right)^{\star}$ such that

$$
\int_{\Omega_{C}} \sigma_{i j}(u) \varepsilon_{i j}(v) d x-\left\langle\lambda, \llbracket v_{i} \rrbracket \nu_{i}\right\rangle_{H_{00}^{1 / 2}\left(\Gamma_{C}\right)^{\star}, H_{00}^{1 / 2}\left(\Gamma_{C}\right)}=\int_{\Gamma_{N}} g_{i} v_{i} d s
$$

for all $v \in H\left(\Omega_{C}\right)$, and

$$
\left\langle\lambda, \llbracket u_{i} \rrbracket \nu_{i}\right\rangle_{H_{00}^{1 / 2}\left(\Gamma_{C}\right)^{\star}, H_{00}^{1 / 2}\left(\Gamma_{C}\right)}=0,
$$

$$
\langle\lambda, \xi\rangle_{H_{00}^{1 / 2}\left(\Gamma_{C}\right)^{\star}, H_{00}^{1 / 2}\left(\Gamma_{C}\right)} \geq 0 \quad \text { for all } 0 \leq \xi \in H_{00}^{1 / 2}\left(\Gamma_{C}\right) .
$$

Applying Green's formula to the first term in (6) we derive for $v \in H\left(\Omega_{C}\right)$ with $v=0$ on $\partial \Omega$ that

$$
\begin{aligned}
\int_{\Omega_{C}} \sigma_{i j}(u) \varepsilon_{i j}(v) d x=-\int_{\Omega_{C}} & \sigma_{i j, j}(u) v_{i} d x \\
& -\left\langle\sigma_{\nu}^{+}(u), v_{i}^{+} \nu_{i}\right\rangle_{1 / 2}+\left\langle\sigma_{\nu}^{-}(u), v_{i}^{-} \nu_{i}\right\rangle_{1 / 2} \\
& -\left\langle\sigma_{\tau i}^{+}(u), v_{\tau i}\right\rangle_{1 / 2}+\left\langle\sigma_{\tau i}^{-}(u), v_{\tau i}^{-}\right\rangle_{1 / 2},
\end{aligned}
$$


where $\langle\cdot, \cdot\rangle_{1 / 2}$ implies the duality pairing between the spaces $H^{1 / 2}\left(\tilde{\Gamma}_{C}\right)$ and $H^{-1 / 2}\left(\tilde{\Gamma}_{C}\right)$. Here the vector $\nu$ also denotes the unit normal vector at the smooth, closed extension $\tilde{\Gamma}_{C}$, and

$$
\sigma_{\nu}(u)=\sigma_{k j}(u) \nu_{j} \nu_{k}, \quad \sigma_{\tau i}(u)=\sigma_{i j}(u) \nu_{j}-\sigma_{\nu}(u) \nu_{i}, \quad v_{\tau i}=v_{i}-\left(v_{k} \nu_{k}\right) \nu_{i}
$$

Utilizing (8) in (6) and (7) we find that

$$
\sigma_{\nu}^{+}(u)=\sigma_{\nu}^{-}(u)=-\lambda, \quad \sigma_{\tau i}^{+}(u)=\sigma_{\tau i}^{-}(u)=0 i=1, \ldots, d \quad \text { on } \Gamma_{C} .
$$

2.2. The semismooth Newton algorithm for penalized problems. For the efficient numerical solution of $(P)$ we propose a semismooth Newton technique applied to a properly regularized version of $(P)$. We show that the algorithm converges at a locally superlinear rate for the continuous problem. Applying the same algorithmic concept to a discretized version of $(P)$, one can establish the local superlinear convergence for each of the discretized problems without the need of regularization.

For $\gamma>0$ consider the problem

$$
\begin{gathered}
\int_{\Omega_{C}} \sigma_{i j}(u) \varepsilon_{i j}(v) d x+\int_{\Gamma_{C}} \mu \llbracket v_{i} \rrbracket \nu_{i} d s=\int_{\Gamma_{N}} g_{i} v_{i} d s \quad \text { for all } v \in H\left(\Omega_{C}\right), \\
\mu=\gamma \min \left(\llbracket u_{i} \rrbracket \nu_{i}, 0\right) .
\end{gathered}
$$

Note that (10) is the first-order optimality condition for the minimization problem

$$
\begin{aligned}
\operatorname{minimize} \frac{1}{2} \int_{\Omega_{C}} & \sigma_{i j}(u) \varepsilon_{i j}(u) d x-\int_{\Gamma_{N}} g_{i} u_{i} d s \\
& +\frac{\gamma}{2} \int_{\Gamma_{C}} \min \left(\llbracket u_{i} \rrbracket \nu_{i}, 0\right)^{2} d s \quad \text { subject to } u \in H\left(\Omega_{C}\right) .
\end{aligned}
$$

Let $u^{\gamma} \in H\left(\Omega_{C}\right)$ denote the unique solution to $\left(P_{\gamma}\right)$, or equivalently to (10), and set $\mu^{\gamma}=\gamma \min \left(\llbracket u_{i}^{\gamma} \rrbracket \nu_{i}, 0\right)$. To solve (10) we apply the following algorithm.

\section{Algorithm 1.}

(0) Choose $u^{(0)} \in H\left(\Omega_{C}\right)$; set $n=0$.

(1) Decompose the crack $\Gamma_{C}$ into sets

$$
I\left(u^{(n)}\right)=\left\{x \in \Gamma_{C}: \quad \llbracket u_{i}^{(n)} \rrbracket \nu_{i} \geq 0\right\}, \quad A\left(u^{(n)}\right)=\Gamma_{C} \backslash I\left(u^{(n)}\right) .
$$

(2) If $n \geq 1$ and $A\left(u^{(n)}\right)=A\left(u^{(n-1)}\right)$ then STOP; else go to step 3.

(3) Compute the solution $u^{(n+1)} \in H\left(\Omega_{C}\right)$ to

$$
\int_{\Omega_{C}} \sigma_{i j}(u) \varepsilon_{i j}(v) d x+\gamma \int_{A\left(u^{(n)}\right)} \llbracket u_{i} \rrbracket \nu_{i} \llbracket v_{j} \rrbracket \nu_{j} d s=\int_{\Gamma_{N}} g_{i} v_{i} d s .
$$


(4) Set

$$
\mu^{(n+1)}= \begin{cases}0 & \text { on } I\left(u^{(n)}\right), \\ \gamma \llbracket u_{i}^{(n+1)} \rrbracket \nu_{i} & \text { on } A\left(u^{(n)}\right) .\end{cases}
$$

(5) Set $n=n+1$ and go to step 1 .

Clearly, step 4 of the algorithm is only introduced for the convenience of notation. Let $\sigma_{\nu}\left(u^{(n)}\right)$ and $\sigma_{\tau}\left(u^{(n)}\right)$ denote the normal and tangential components of the stress tensor at the crack $\Gamma_{C}$ depending on the solution $u^{(n)}$. From (8) and (11) we find that for $n=1,2, \ldots$

$$
\begin{gathered}
\sigma_{\tau i}^{ \pm}\left(u^{(n)}\right)=0 \quad i=1, \ldots, d, \quad \sigma_{\nu}^{+}\left(u^{(n)}\right)=\sigma_{\nu}^{-}\left(u^{(n)}\right) \quad \text { on } \Gamma_{C}, \\
\sigma_{\nu}^{ \pm}\left(u^{(n)}\right)=0 \quad \text { on } I\left(u^{(n)}\right), \quad \sigma_{\nu}^{ \pm}\left(u^{(n)}\right)=\gamma \llbracket u_{i}^{(n)} \rrbracket \nu_{i} \quad \text { on } A\left(u^{(n)}\right) .
\end{gathered}
$$

We next analyze the convergence of $\left(u^{\gamma}, \mu^{\gamma}\right)$ to $\left(u, \sigma_{\nu}^{ \pm}(u)\right)$ as $\gamma \rightarrow \infty$, where $u$ denotes the solution to $(P)$, and the convergence of $\left(u^{(n)}, \mu^{(n)}\right)$ to $\left(u^{\gamma}, \mu^{\gamma}\right)$ as $n \rightarrow \infty$.

Proposition 2.1. For $\gamma \rightarrow \infty$ we have $u^{\gamma} \rightarrow u$ strongly in $H\left(\Omega_{C}\right)$ and $\mu^{\gamma} \rightarrow \sigma_{\nu}^{ \pm}(u)$ weakly in $H_{00}^{1 / 2}\left(\Gamma_{C}\right)^{\star}$.

Proof. Strong convergence of $u^{\gamma}$ to $u$ in $H\left(\Omega_{C}\right)$ is proved in [10, pp. 161]. From (10) it follows that $\mu^{\gamma}$ is bounded in $H_{00}^{1 / 2}\left(\Gamma_{C}\right)^{\star}$ as $\gamma>0$. Hence there exists $\mu \in H_{00}^{1 / 2}\left(\Gamma_{C}\right)^{\star}$ such that $\mu^{\gamma} \rightarrow \mu$ weakly in $H_{00}^{1 / 2}\left(\Gamma_{C}\right)^{\star}$ for $\gamma \rightarrow \infty$. Taking the limit $\gamma \rightarrow \infty$ in (10) with $u=u^{\gamma}, \mu=\mu^{\gamma}$ we find

$$
\int_{\Omega_{C}} \sigma_{i j}(u) \varepsilon_{i j}(v) d x+\left\langle\mu, \llbracket v_{i} \rrbracket \nu_{i}\right\rangle_{H_{00}^{1 / 2}\left(\Gamma_{C}\right)^{\star}, H_{00}^{1 / 2}\left(\Gamma_{C}\right)}=\int_{\Gamma_{N}} g_{i} v_{i} d s .
$$

For $\lambda=-\mu$ this equation coincides with (6). Hence, due to (9) $\mu^{\gamma}$ converges weakly in $H_{00}^{1 / 2}\left(\Gamma_{C}\right)^{\star}$ to $\sigma_{\nu}^{ \pm}(u)=-\lambda$.

Next we address the convergence $u^{(n)} \rightarrow u^{\gamma}$ with $\gamma>0$. Note that for given $\mu \in L^{2}\left(\Gamma_{C}\right)$ equation (10a) is the variational formulation of the boundary value problem

$$
\begin{array}{ll} 
& -\sigma_{i j, j}(u)=0 \quad \text { in } \Omega_{C}, \\
(\operatorname{BVP}(\mu, g)) \quad & u=0 \quad \text { on } \Gamma_{D}, \quad \sigma_{i j}(u) \eta_{j}=g_{i} \quad i=1, \ldots, d \quad \text { on } \Gamma_{N}, \\
& \sigma_{\nu}^{ \pm}(u)=\mu, \quad \sigma_{\tau i}^{ \pm}(u)=0 \quad i=1, \ldots, d \quad \text { on } \Gamma_{C},
\end{array}
$$

for $\eta=\left(\eta_{1}, \ldots, \eta_{d}\right)^{\top}$ being the outward unit normal vector to $\partial \Omega$. By Korn's inequality there exists a unique solution $u=u(\mu) \in H\left(\Omega_{C}\right)$ to $(\operatorname{BVP}(\mu, g))$. Let the mapping $\Psi: L^{2}\left(\Gamma_{C}\right) \rightarrow L^{2}\left(\Gamma_{C}\right)$ be defined by

$$
\Psi(\mu)=\gamma \min \left(\llbracket u_{i}(\mu) \rrbracket \nu_{i}, 0\right)-\mu \text {. }
$$

Then problem (10) can be expressed as the nonlinear and nondifferentiable equation in $L^{2}\left(\Gamma_{C}\right)$ :

$$
\Psi(\mu)=0
$$


Let $\chi$ be the characteristic function corresponding to the min-operator, i.e.,

$$
\chi(s)= \begin{cases}0 & \text { if } s \geq 0 \\ 1 & \text { if } s<0 .\end{cases}
$$

A semismooth Newton step to (13) is given by

$$
-\delta \mu+\gamma \chi\left(\llbracket u_{i}\left(\mu^{(n)}\right) \rrbracket \nu_{i}\right) \llbracket \hat{u}_{j}(\delta \mu) \rrbracket \nu_{j}=-\gamma \min \left(\llbracket u_{i}\left(\mu^{(n)}\right) \rrbracket \nu_{i}, 0\right)+\mu^{(n)},
$$

where $\hat{u}(\delta \mu)$ denotes the variational solution to $(\operatorname{BVP}(\delta \mu, 0))$. From (14) we deduce for $\mu^{(n+1)}=\mu^{(n)}+\delta \mu$ that

$$
\mu^{(n+1)}= \begin{cases}0 & \text { on } I\left(u\left(\mu^{(n)}\right)\right), \\ \gamma \llbracket u_{i}\left(\mu^{(n)}\right)+\hat{u}_{i}(\delta \mu) \rrbracket \nu_{i}=\gamma \llbracket u_{i}\left(\mu^{(n+1)}\right) \rrbracket \nu_{i} & \text { on } A\left(u\left(\mu^{(n)}\right)\right),\end{cases}
$$

where $u\left(\mu^{(n+1)}\right)$ solves (11). Hence Algorithm 1 describes a semismooth Newton step applied to (13).

Theorem 2.1. If $\left\|u^{(0)}-u^{\gamma}\right\|_{H^{1}\left(\Omega_{C}\right)^{d}}$ is sufficiently small, then the sequence $\left(\left(u^{(n)}, \mu^{(n)}\right)\right)$ generated by Algorithm 1 converges superlinearly to $\left(u^{\gamma}, \mu^{\gamma}\right)$ in $H^{1}\left(\Omega_{C}\right)^{d} \times L^{2}\left(\Gamma_{C}\right)$.

Proof. Let us set $\overline{\delta u}=u^{(n+1)}-u^{\gamma}, \overline{\delta \mu}=\mu^{(n+1)}-\mu^{\gamma}$. From (10) and (11) we have

$$
\int_{\Omega_{C}} \sigma_{i j}(\overline{\delta u}) \varepsilon_{i j}(v) d x=-\int_{\Gamma_{C}} \overline{\delta \mu} \llbracket v_{i} \rrbracket \nu_{i} d s \quad \text { for } v \in H\left(\Omega_{C}\right) .
$$

Note that

$$
\begin{aligned}
\overline{\delta \mu}= & \gamma \min \left(\llbracket u_{i}^{(n)} \rrbracket \nu_{i}, 0\right)+\gamma \chi\left(\llbracket u_{i}^{(n)} \rrbracket \nu_{i}\right)\left(\llbracket u_{j}^{(n+1)}-u_{j}^{(n)} \rrbracket \nu_{j}\right)-\gamma \min \left(\llbracket u_{i}^{\gamma} \rrbracket \nu_{i}, 0\right) \\
= & \gamma \min \left(\llbracket u_{i}^{\gamma} \rrbracket \nu_{i}+\llbracket u_{i}^{(n)}-u_{i}^{\gamma} \rrbracket \nu_{i}, 0\right)-\gamma \min \left(\llbracket u_{i}^{\gamma} \rrbracket \nu_{i}, 0\right) \\
& \quad-\gamma \chi\left(\llbracket u_{i}^{\gamma} \rrbracket \nu_{i}+\llbracket u_{i}^{(n)}-u_{i}^{\gamma} \rrbracket \nu_{i}\right) \llbracket u_{j}^{(n)}-u_{j}^{\gamma} \rrbracket \nu_{j}+\gamma \chi\left(\llbracket u_{i}^{(n)} \rrbracket \nu_{i}\right) \llbracket \bar{\delta} u_{j} \rrbracket \nu_{j} \\
= & : R+\gamma \chi\left(\llbracket u_{i}^{(n)} \rrbracket \nu_{i}\right) \llbracket \overline{\delta u}_{j} \rrbracket \nu_{j} .
\end{aligned}
$$

Let $p>2$. For every $v \in L^{p}\left(\Gamma_{C}\right)$ we have

$$
\|\min (v+h, 0)-\min (v, 0)-\chi(v+h) h\|_{L^{2}\left(\Gamma_{C}\right)}=o\left(\|h\|_{L^{p}\left(\Gamma_{C}\right)}\right)
$$

as $\|h\|_{L^{p}\left(\Gamma_{C}\right)} \rightarrow 0$. This is proved for domains in $\mathbb{R}^{d}$ in $[4$, Appendix A], and the proof there can easily be modified to the case of functions on $\Gamma_{C}$. Recall that in dimension $d>1$ the trace of $H^{1}\left(\Omega_{C}\right)$ onto $L^{p}\left(\Gamma_{C}\right)$ with $p=\frac{2 d}{d-1}$ is continuous. In our case $p=4$ for $d=2$, or $p=3$ as $d=3$. Applying (16) with such $p$ to $\mathrm{R}$ we find that

$$
\|R\|_{L^{2}\left(\Gamma_{C}\right)}=o\left(\left\|\llbracket u_{i}^{(n)}-u_{i}^{\gamma} \rrbracket \nu_{i}\right\|_{L^{p}\left(\Gamma_{C}\right)}\right),
$$

and consequently

$$
\|R\|_{L^{2}\left(\Gamma_{C}\right)}=o\left(\left\|u^{(n)}-u^{\gamma}\right\|_{H^{1}\left(\Omega_{C}\right)^{d}}\right)
$$


Choosing $v=\overline{\delta u}$ in (15) and using Korn's inequality implies that

$$
c_{0}\|\overline{\delta u}\|_{H^{1}\left(\Omega_{C}\right)^{d}}^{2} \leq\|R\|_{L^{2}\left(\Gamma_{C}\right)}\|\overline{\delta u}\|_{L^{2}\left(\Gamma_{C}\right)}
$$

for some constant $c_{0}>0$. Hence, from (17) it follows

$$
\|\overline{\delta u}\|_{H^{1}\left(\Omega_{C}\right)^{d}}=o\left(\left\|u^{(n)}-u^{\gamma}\right\|_{H^{1}\left(\Omega_{C}\right)^{d}}\right)
$$

and consequently the final estimate

$$
\left\|u^{(n+1)}-u^{\gamma}\right\|_{H^{1}\left(\Omega_{C}\right)^{d}}+\left\|\mu^{(n+1)}-\mu^{\gamma}\right\|_{L^{2}\left(\Gamma_{C}\right)}=o\left(\left\|u^{(n)}-u^{\gamma}\right\|_{H^{1}\left(\Omega_{C}\right)^{d}}\right) .
$$

\section{The Semismooth Newton method for the Discretized PROBLEMS}

Let us consider a discretization of $(P)$ which results in a quadratic programming problem of the type

$\left(P_{N}\right) \quad$ minimize $\frac{1}{2} u^{\top} L u-f^{\top} u$ over $u \in \mathbb{R}^{N}$ subject to $\Lambda u \geq 0$,

where $L \in \mathbb{R}^{N \times N}$ is positive-definite and symmetric, $f \in \mathbb{R}^{N}$, and $\Lambda \in$ $\mathbb{R}^{|B| \times N}$. Here, for the index set $B \subset\{1, \ldots, N\}$ we denote by $|B|$ its cardinality. Before we give a detailed description of $\Lambda$, we define the index set $\mathcal{B} \subset B \times B$. Each pair $\left(i^{+}, i^{-}\right) \in \mathcal{B}$ is induced by an index $i \in B$, which belongs to a nodal point at the crack $\Gamma_{C}$, i.e., $i^{+}=i^{+}(i)$ and $i^{-}=i^{-}(i)$. Thus, $|\mathcal{B}|=|B|$. This definition allows us to write the discrete non-penetration condition as

$$
\left(u_{k}^{o}\right)_{i^{+}}\left(\nu_{k}\right)_{i^{+}}+\left(u_{k}^{o}\right)_{i^{-}}\left(\nu_{k}\right)_{i^{-}} \geq 0 \quad \text { for all }\left(i^{+}, i^{-}\right) \in \mathcal{B}, k=1, \ldots, d,
$$

where each vector $\left(u^{o}\right)_{i^{ \pm}}$is given by $\left(u^{o}\right)_{i^{ \pm}}=\left(u_{1}, \ldots, u_{d}\right)_{i^{ \pm}}$, which are the displacement vectors at the nodal points on $\Gamma_{C}^{+}$and $\Gamma_{C}^{-}$, respectively, with associated index $i$. Note that we use summation over repeated indices $k$ in (19). Due to our convention concerning the normal vector along the crack, we have $\left(\nu_{k}\right)_{i^{+}}=\left(\nu_{k}\right)_{i}=-\left(\nu_{k}\right)_{i^{-}}$. Now let us assume that the vector $u \in \mathbb{R}^{N}$ is partitioned into $u=\left(u_{D}, u_{\tilde{B}}\right)^{\top}$ where the index set $\tilde{B}$ and the vector $u_{\tilde{B}}$ are obtained from the following description: Let $u_{k}^{B^{+}}=$ $\left(\left(u_{k}^{o}\right)_{i^{+}(1)}, \ldots,\left(u_{k}^{o}\right)_{i^{+}(B)}\right) \in \mathbb{R}_{|B|}$ for $k=1, \ldots, d$, and analogously for $u_{k}^{B^{-}}$. Then

$$
u_{\tilde{B}}=\left(u_{1}^{B^{+}}, \ldots, u_{d}^{B^{+}}, u_{1}^{B^{-}}, \ldots, u_{d}^{B^{-}}\right)^{\top} \in \mathbb{R}^{2 d|B|} .
$$

Thus, we infer that $|\tilde{B}|=2 d|B|$. Now, the matrix $\Lambda$ is defined by

$$
\begin{aligned}
\Lambda & =\left(\mathbf{0}, T_{1}, \ldots, T_{d},-T_{1}, \ldots,-T_{d}\right), \\
T_{k} & =\operatorname{diag}\left(\left(\nu_{k}\right)_{1}, \ldots,\left(\nu_{k}\right)_{|B|}\right) \in \mathbb{R}^{|B| \times|B|} \text { for } k=1, \ldots, d,
\end{aligned}
$$

where $\mathbf{0}$ is the $|B| \times|D|$-zero matrix with $|D|=N-|\tilde{B}|$. Therefore $\Lambda$ is related to the discretized non-penetration condition. For the above description of $\Lambda$ to be valid we throughout assume that the nodal values at $\Gamma_{C}^{ \pm}$are ordered as outlined in (20). 
Note that the column-rank of $\Lambda$ is $|B|$, and for later use we also observe that

$$
\operatorname{ker}\left(\Lambda L^{-1} \Lambda^{\top}\right)=\left\{w \in \mathbb{R}^{|B|}: \Lambda L^{-1} \Lambda^{\top} w=0\right\}=\{0\} .
$$

Further we have that $\Lambda L^{-1} \Lambda^{\top}$ is positive definite.

It is well-known that the solution $u^{*} \in \mathbb{R}^{N}$ of problem $\left(P_{N}\right)$ is characterized as the solution to the variational inequality problem: Find $u \in \mathbb{R}^{N}$ such that

$$
\Lambda u \geq 0, \quad(f-L u)^{\top}(v-u) \leq 0 \quad \text { for all } v \in \mathbb{R}^{N} \text { with } \Lambda v \geq 0 .
$$

Introducing a Lagrange multiplier $\lambda \in \mathbb{R}^{|B|}$ this problem can be equivalently written as: Find $(u, \lambda) \in \mathbb{R}^{N} \times \mathbb{R}^{|B|}$ such that

$$
\begin{gathered}
L u-\Lambda^{\top} \lambda=f, \\
\Phi(u, \lambda)=\max (\alpha \lambda-\Lambda u, 0)-\alpha \lambda=0,
\end{gathered}
$$

where $\alpha>0$ is an arbitrarily fixed real. Note that (22), respectively (23), is also sufficient for $u$ to be the solution of $\left(P_{N}\right)$, i.e., $u^{*}=u$. By $\lambda^{*}$ we shall denote the Lagrange multiplier associated with $u^{*}$.

3.1. The active set perspective and fast local convergence. In order to devise our semismooth Newton method for solving $\left(P_{N}\right)$ we focus on $(23)$. Setting $y:=(u, \lambda)^{\top} \in \mathbb{R}^{N} \times \mathbb{R}^{|B|}$ we restate the system (23) as

$$
F(y)=\left(\begin{array}{c}
L u-\Lambda^{\top} \lambda-f \\
\Phi(u, \lambda)
\end{array}\right)=0 .
$$

with the non-differentiable function $F: \mathbb{R}^{N+|B|} \rightarrow \mathbb{R}^{N+|B|}$. Our aim now is to show that $F$ is slantly differentiable. For this purpose we introduce the matrix $\chi_{S} \in \mathbb{R}^{|B| \times|B|}$ as

$$
\chi_{S}=\operatorname{diag}\left(s_{1}, \ldots, s_{|B|}\right), \quad \text { with } s_{i}= \begin{cases}1 & \text { if } i \in S, \\ 0 & \text { if } i \notin S,\end{cases}
$$

and we define

$$
\begin{aligned}
& A(y)=\left\{i \in B: \alpha \lambda_{i}-(\Lambda u)_{i}>0\right\}, \\
& I(y)=\left\{i \in B: \alpha \lambda_{i}-(\Lambda u)_{i} \leq 0\right\} .
\end{aligned}
$$

These definitions imply $\chi_{A(y)}+\chi_{I(y)}=1$ which allows us to rewrite the function $\Phi$ in (23b) in the form

$$
\Phi(u, \lambda)=-\chi_{A(y)} \Lambda u-\alpha \chi_{I(y)} \lambda .
$$

As a consequence, $F$ in (24) admits the representation

$$
F(y)=G(y) y-\left(\begin{array}{l}
f \\
0
\end{array}\right) \text { with } G(y)=\left(\begin{array}{lr}
L & -\Lambda^{\top} \\
-\chi_{A(y)} \Lambda & -\alpha \chi_{I(y)}
\end{array}\right) .
$$


Now it can be easily verified that

$$
\begin{aligned}
F(y+h) & -F(y)-G(y+h) h \\
& =\left(\begin{array}{c}
0 \\
-\left(\chi_{A(y+h)}-\chi_{A(y)}\right) \Lambda u-\alpha\left(\chi_{I(y+h)}-\chi_{I(y)}\right) \lambda
\end{array}\right)
\end{aligned}
$$

and that the right hand side above is zero for all $h \in \mathbb{R}^{N} \times \mathbb{R}^{|B|}$ satisfying

$$
\|h\|_{\infty}<m / 2 \max (\|\Lambda\|, \alpha)
$$

with $m$ given by

$$
m=\min \left\{\left|\alpha \lambda_{i}-(\Lambda u)_{i}\right|: i \in B, \alpha \lambda_{i}-(\Lambda u)_{i} \neq 0\right\} .
$$

Thus, $F$ satisfies

$$
\lim _{h \rightarrow 0} \frac{1}{\|h\|}\|F(y+h)-F(y)-G(y+h) h\|=0,
$$

and, according to (4), $G$ serves as a generalized derivative of the nondifferentiable mapping $F$.

Remark 3.1. The set $A\left(y^{*}\right)$ is called the active set at $y^{*}$ since $\lambda_{i}^{*}>0$ and $\left(\Lambda u^{*}\right)_{i}=0$ for all $i \in A\left(y^{*}\right)$ at the solution $y^{*}$ of (23). On the other hand, for $i \in I\left(y^{*}\right)$ we have $\lambda_{i}^{*}=0$ and $\left(\Lambda u^{*}\right)_{i} \geq 0$. Therefore we call $I\left(y^{*}\right)$ the inactive set at $y^{*}$.

Based on the above considerations we can now define the semismooth Newton method for computing the solution to (24): For some initial guess $y^{(0)}$ compute

$$
y^{(n+1)}=y^{(n)}-G\left(y^{(n)}\right)^{-1} F\left(y^{(n)}\right), \quad n=0,1, \ldots
$$

The local convergence behavior of this iteration is the content of the following theorem.

Theorem 3.1. The semismooth Newton iteration (27) is well-defined, and the sequence of iterates $\left(y^{(n)}\right)$ converges superlinearly to $y^{*}$ with $F\left(y^{*}\right)=0$ provided that $y^{(0)}$ is sufficiently close to $y^{*}$.

Proof. We first show that $G(y)$ is a bijection for every $y \in \mathbb{R}^{N}$. For arbitrary $g=\left(g_{u}, g_{\lambda}\right)^{\top} \in \mathbb{R}^{N} \times \mathbb{R}^{|B|}$ we verify that there exists a unique solution $z=\left(z_{u}, z_{\lambda}\right)^{\top} \in \mathbb{R}^{N} \times \mathbb{R}^{|B|}$ such that $G(y) z=g$, i.e.,

$$
\begin{gathered}
L z_{u}-\Lambda^{\top} z_{\lambda}=g_{u}, \\
-\chi_{A(y)} \Lambda z_{u}-\alpha \chi_{I(y)} z_{\lambda}=g_{\lambda} .
\end{gathered}
$$

Since $L$ is positive definite and, hence, invertible we obtain

$$
z_{u}=L^{-1} \Lambda^{\top} z_{\lambda}+L^{-1} g_{u}
$$

Using this relation in $(28 \mathrm{~b})$ results in

$$
\left(\chi_{A(y)} \Lambda L^{-1} \Lambda^{\top}+\alpha \chi_{I(y)}\right) z_{\lambda}=-\chi_{A(y)} \Lambda L^{-1} g_{u}-g_{\lambda}=: \tilde{g} .
$$


As noted earlier, the $|B| \times|B|$-matrix $M:=\Lambda L^{-1} \Lambda^{\top}$ is positive definite. Whence (30) yields

$$
\begin{gathered}
\left(z_{\lambda}\right)_{A(y)}=\left(M_{A(y) A(y)}\right)^{-1} \tilde{g}_{A(y)}-\frac{1}{\alpha}\left(M_{A(y) A(y)}\right)^{-1} M_{A(y) I(y)} \tilde{g}_{I(y)}, \\
\left(z_{\lambda}\right)_{I(y)}=\frac{1}{\alpha} \tilde{g}_{I(y)},
\end{gathered}
$$

where we use the partition of the matrix $M$ and the involved vectors into blocks

$$
M=\left(\begin{array}{ll}
M_{A(y) A(y)} & M_{A(y) I(y)} \\
M_{I(y) A(y)} & M_{I(y) I(y)}
\end{array}\right), \quad z_{\lambda}=\left(\begin{array}{c}
\left(z_{\lambda}\right)_{A(y)} \\
\left(z_{\lambda}\right)_{I(y)}
\end{array}\right), \quad \tilde{g}=\left(\begin{array}{c}
\tilde{g}_{A(y)} \\
\tilde{g}_{I(y)}
\end{array}\right) .
$$

This proves that $(27)$ is well-defined for every $n \in \mathbb{N}_{0}$. Moreover, from (31) and (29) we infer that $\left\|G\left(y^{(n)}\right)^{-1}\right\| \leq C$ for some constant $C>0$ independent of $n$.

The local superlinear convergence now follows from standard results; see, e.g., [4, Theorem 1.1].

In our numerical implementation we realize the semismooth Newton iteration (27) in the following way.

\section{Algorithm 2.}

(0) Choose $y^{(0)}=\left(u^{(0)}, \lambda^{(0)}\right) \in \mathbb{R}^{N} \times \mathbb{R}^{|B|}$; set $n=0$.

(1) Compute the active and inactive sets at $y^{(n)}$ :

$$
\begin{aligned}
& A\left(y^{(n)}\right)=\left\{i \in B: \alpha \lambda_{i}^{(n)}-\left(\Lambda u^{(n)}\right)_{i}>0\right\} . \\
& I\left(y^{(n)}\right)=\left\{i \in B: \alpha \lambda_{i}^{(n)}-\left(\Lambda u^{(n)}\right)_{i} \leq 0\right\} .
\end{aligned}
$$

(2) If $n \geq 1$ and $A\left(y^{(n)}\right)=A\left(y^{(n-1)}\right)$ then STOP; else go to step 3 .

(3) Solve for $y^{(n+1)}=\left(u^{(n+1)}, \lambda^{(n+1)}\right) \in \mathbb{R}^{N} \times \mathbb{R}^{|B|}$ :

$$
L u^{(n+1)}-\Lambda^{\top} \lambda^{(n+1)}=f
$$

$$
\left(\Lambda u^{(n+1)}\right)_{i}=0 \text { for all } i \in A\left(y^{(n)}\right), \quad \lambda_{i}^{(n+1)}=0 \text { for all } i \in I\left(y^{(n)}\right) .
$$

(4) Set $n=n+1$ and go to step 1 .

Note that Algorithm 2 and the Newton process (27) are equivalent. In order to see this, we rewrite $(27)$ with respect to $y^{(n+1)}-y^{(n)}$ as

$$
\begin{array}{r}
L\left(u^{(n+1)}-u^{(n)}\right)-\Lambda^{\top}\left(\lambda^{(n+1)}-\lambda^{(n)}\right)=f-L u^{(n)}+\Lambda^{\top} \lambda^{(n)}, \\
-\chi_{A\left(y^{(n)}\right)} \Lambda\left(u^{(n+1)}-u^{(n)}\right)-\alpha \chi_{I\left(y^{(n)}\right)}\left(\lambda^{(n+1)}-\lambda^{(n)}\right) \\
=\alpha \lambda^{(n)}-\max \left(\alpha \lambda^{(n)}-\Lambda u^{(n)}, 0\right) .
\end{array}
$$

Equation (34a) implies (33a), and the choices (32) and (33b) realize the semismooth Newton step for the nonsmooth equation (34b). In fact, for $i \in I\left(y^{(n)}\right)$ we have $-\left(\Lambda u^{(n)}\right)_{i}+\alpha \lambda_{i}^{(n)} \leq 0$. Thus, (34b) yields $\lambda_{i}^{(n+1)}=0$ for all $i \in I\left(y^{(n)}\right)$. For all $i \in A\left(y^{(n)}\right)$ we have $-\left(\Lambda u^{(n)}\right)_{i}+\alpha \lambda_{i}^{(n)}>0$. Hence, 
(34) implies $\left(\Lambda u^{(n+1)}\right)_{i}=0$ for all $i \in A\left(y^{(n)}\right)$. Combining the two cases we recover (33b). As a consequence problem (33) is well-defined and, according to Theorem 3.1, it admits a unique solution.

Due to step 1 and our interpretation of $A\left(y^{(n)}\right)$ and $I\left(y^{(n)}\right)$ as the active and inactive sets at $y^{(n)}$ (cf. Remark 3.1), Algorithm 2 resembles an activeset-type method; see [4] for more details on this aspect.

The stopping rule in step 2 of Algorithm 2 is motivated by the following considerations. For $i \in A\left(y^{(n-1)}\right)$ we have $\left(\Lambda u^{(n)}\right)_{i}=0$, and for $i \in I\left(y^{(n-1)}\right)$ we obtain $\lambda_{i}^{(n)}=0$. Hence, if we assume that $A\left(y^{(n-1)}\right)=A\left(y^{(n)}\right)$, then from (32a) we infer $\lambda_{i}^{(n)}>0$ for all $i \in A\left(y^{(n)}\right)$, and $\left(\Lambda u^{(n)}\right)_{i} \geq 0$ for all $i \in I\left(y^{(n)}\right)$ by (32b). This, together with (33a), proves that the iterate $y^{(n)}=$ $\left(u^{(n)}, \lambda^{(n)}\right)$ upon termination of Algorithm 2 in step 2 satisfies $F\left(y^{(n)}\right)=0$.

Let us emphasize that the successful termination occurs after a finite number of iterations. Indeed, since there exists only a finite number of choices for $A\left(y^{(n)}\right)$ with $A\left(y^{(n)}\right) \neq A\left(y^{(m)}\right)$ for $m \neq n$ (and analogously for $\left.I\left(y^{(n)}\right)\right)$, Theorem 3.1 yields the finite step convergence.

3.2. Reduced problems. We start by considering the reduced version of (23) given by

$$
\begin{aligned}
\hat{L} \hat{u}-\hat{f}-\hat{\Lambda}^{\top} \hat{\lambda} & =0, \\
\hat{\Phi}(\hat{u}, \hat{\lambda}):=\max (-\hat{\Lambda} \hat{u}+\alpha \hat{\lambda}, 0)-\alpha \hat{\lambda} & =0 .
\end{aligned}
$$

Here we use

$$
\hat{L}=L_{\tilde{B} \tilde{B}}-L_{\tilde{B} D} L_{D D}^{-1} L_{D \tilde{B}} \in \mathbb{R}^{|\tilde{B}| \times|\tilde{B}|}, \quad \hat{f}=f_{\tilde{B}}-L_{\tilde{B} D} L_{D D}^{-1} f_{D} \in \mathbb{R}^{|\tilde{B}|}
$$

and further

$$
\hat{\Lambda}=\left(T_{1}, \ldots, T_{d},-T_{1}, \ldots,-T_{d}\right) \in \mathbb{R}^{|B| \times|\tilde{B}|}, \quad \hat{\lambda} \in \mathbb{R}^{|B|} .
$$

In fact, (35) is obtained from (23) utilizing the relation $u_{D}=L_{D D}^{-1}\left(f_{D}-\right.$ $\left.L_{D \tilde{B}} u_{\tilde{B}}\right)$ which is due to (23a), the partition of $L, f$, and $u$ according to $D, \tilde{B}$, and $\Lambda=(\mathbf{0}, \hat{\Lambda})$.

Note that $\hat{L}$ denotes the Schur complement of $L_{D D}$ in $L$. Since $L$ is symmetric and positive definite, also the Schur complement $\hat{L}$ is symmetric and positive definite; see [17]. As a consequence, (35) admits a unique solution $\hat{u}^{*}$ with associated Lagrange multiplier $\hat{\lambda}^{*}$, and it represents the first order necessary and sufficient condition of

$$
\text { minimize } \frac{1}{2} \hat{u}^{\top} \hat{L} \hat{u}-\hat{f}^{\top} \hat{u} \text { over } \hat{u} \in \mathbb{R}^{|\tilde{B}|} \text { subject to } \hat{\Lambda} \hat{u} \geq 0 .
$$

The relation between $\left(P_{N}\right)$ and $(36)$ is investigated next.

Proposition 3.1. $\quad$ (a) Let $u^{*}=\left(u_{D}^{*}, u_{\tilde{B}}^{*}\right) \in \mathbb{R}^{N}$ be the optimal solution of $\left(P_{N}\right)$ with optimal multiplier $\lambda^{*}$. Then $\hat{u}^{*}=u_{\tilde{B}}^{*}$, i.e., $u_{\tilde{B}}^{*}$ solves the reduced problem (36). The corresponding multiplier is $\hat{\lambda}^{*}=\lambda^{*}$. 
(b) Let $\hat{u}^{*}$ denote the solution of (36) with multiplier $\hat{\lambda}^{*}$. Then $\left(\bar{u}_{D}^{*}, \hat{u}^{*}\right)$ with $\bar{u}_{D}^{*}=L_{D D}^{-1}\left(f_{D}-L_{D \tilde{B}} \hat{u}^{*}\right)$ solves $\left(P_{N}\right)$ with associated multiplier $\hat{\lambda}^{*}$.

Proof. (a) The optimal $u^{*}$ satisfies (23). Thus, after partitioning (23a) according to $D$ and $\tilde{B}$, we have $u_{D}^{*}=L_{D D}^{-1}\left(f_{D}-L_{D \tilde{B}} u_{\tilde{B}}^{*}\right)$. Inserting this in $L_{\tilde{B} \tilde{B}} u_{\tilde{B}}^{*}+L_{\tilde{B} D} u_{D}^{*}-f_{\tilde{B}}-\left(\Lambda^{\top}\right)_{\tilde{B}} \cdot \lambda^{*}=0$ yields $\hat{L} u_{\tilde{B}}^{*}-\hat{f}-\hat{\Lambda}^{\top} \lambda^{*}=0$. One easily verifies that $\max \left(-\Lambda_{\cdot \tilde{B}} u_{\tilde{B}}^{*}+\alpha \lambda^{*}, 0\right)-\alpha \lambda^{*}=0$. Since the solution of (36), and hence (35), is unique we infer $\hat{\lambda}^{*}=\lambda^{*}, \hat{u}^{*}=u_{\tilde{B}}^{*}$ and assertion (a) follows.

(b) The assertion essentially follows from reverting the arguments in (a).

We may also devise a semismooth Newton method of the type of Algorithm 2 for iteratively solving (35). In this case the analogue of step 2 consists in finding the sets

$$
\begin{aligned}
\hat{A}\left(\hat{y}^{(n)}\right) & =\left\{i \in B: \alpha \hat{\lambda}_{i}^{(n)}-\left(\hat{\Lambda} \hat{u}^{(n)}\right)_{i}>0\right\} \\
\hat{I}\left(\hat{y}^{(n)}\right) & =\left\{i \in B: \alpha \hat{\lambda}_{i}^{(n)}-\left(\hat{\Lambda} \hat{u}^{(n)}\right)_{i} \leq 0\right\}
\end{aligned}
$$

with $\hat{y}^{(n)}:=\left(\hat{u}^{(n)}, \hat{\lambda}^{(n)}\right)$. Accordingly, in the new step 3 we determine $\left(\hat{u}^{(n+1)}, \hat{\lambda}^{(n+1)}\right)$ such that

$$
\begin{aligned}
& \hat{L} \hat{u}^{(n+1)}-\hat{\Lambda}^{\top} \hat{\lambda}^{(n+1)}=\hat{f}, \\
& \left(\hat{\Lambda} \hat{u}^{(n+1)}\right)_{i}=0 \text { for all } i \in \hat{A}\left(\hat{u}^{(n)}\right), \quad \hat{\lambda}_{i}^{(n+1)}=0 \text { for all } i \in \hat{I}\left(\hat{u}^{(n)}\right) .
\end{aligned}
$$

The primal iterate on $D$ is computed from $u_{D}^{(n+1)}=L_{D D}^{-1}\left(f_{D}-L_{D} \tilde{B}^{(n+1)}\right)$. Then it is straightforward to verify that if $\hat{u}^{(0)}=u_{\tilde{B}}^{(0)}$ and $\hat{\lambda}^{(0)}=\lambda^{(0)}$ then both algorithms produce the same sequence of primal and dual iterates.

Next we establish the following property of the matrix $\hat{\Lambda} \hat{L}^{-1} \hat{\Lambda}^{\top} \in \mathbb{R}^{|B| \times|B|}$.

Proposition 3.2. The matrix $\hat{\Lambda} \hat{L}^{-1} \hat{\Lambda}^{\top}$ is positive definite.

Proof. Since $\hat{\Lambda}^{\top}$ has full column rank, we have $\operatorname{ker}\left(\hat{\Lambda}^{\top}\right)=\{0\}$. Thus, for $\hat{z} \neq 0$ we have $\hat{\Lambda}^{\top} \hat{z} \neq 0$. From the fact that $\hat{L}$ is positive definite we conclude

$$
\hat{z}^{\top} \hat{\Lambda} \hat{L}^{-1} \hat{\Lambda}^{\top} \hat{z}>0 \quad \text { for all } \hat{z} \in \mathbb{R}^{|B|} \backslash\{0\} .
$$

Now, multiplying (35a) first by $\hat{L}^{-1}$ and then by $\hat{\Lambda}$, we obtain

$$
\hat{\Lambda} \hat{u}-\hat{\Lambda} \hat{L}^{-1} \hat{f}-\left(\hat{\Lambda} \hat{L}^{-1} \hat{\Lambda}^{\top}\right) \hat{\lambda}=0 .
$$

For convenience we define $\hat{M}=\left(\hat{\Lambda} \hat{L}^{-1} \hat{\Lambda}^{\top}\right)^{-1}$. Setting $\hat{v}:=\hat{\Lambda} \hat{u} \in \mathbb{R}^{|B|}$, multiplying (37) by $\hat{M}$ and taking (35b) into account, we arrive at the 
linear complementarity problem: Find $\hat{v} \in \mathbb{R}^{|B|}$ such that

$$
\begin{aligned}
\hat{M} \hat{v}-\hat{q} & \geq 0, \\
\hat{v} & \geq 0, \\
\hat{v}^{\top}(\hat{M} \hat{v}-\hat{q}) & =0,
\end{aligned}
$$

where $\hat{q}:=\hat{M} \hat{\Lambda} \hat{L}^{-1} \hat{f} \in \mathbb{R}^{|B|}$. It is the first order necessary and sufficient condition of the strictly convex quadratic optimization problem

$$
\text { minimize } \frac{1}{2} \hat{v}^{\top} \hat{M} \hat{v}-\hat{q}^{\top} \hat{v} \quad \text { over } \hat{v} \in \mathbb{R}^{|B|} \text { subject to } \hat{v} \geq 0,
$$

which admits a unique solution $\hat{v}^{*}$. Let $\hat{\mu}^{*}$ denote the associated optimal multiplier.

We have the following relation between the reduced problems (36) and (39).

Proposition 3.3. $\quad$ (a) Let $\hat{u}^{*}$ be the solution to (36) with multiplier $\hat{\lambda}^{*}$. Then $\hat{v}^{*}=\hat{\Lambda} \hat{u}^{*}$ with multiplier $\hat{\mu}^{*}=\hat{\lambda}^{*}$ solves (39).

(b) Let $\hat{v}^{*}$ be the solution to (39) with multiplier $\hat{\mu}^{*}$. Then $\hat{v}^{*}=\hat{\Lambda} \hat{u}^{*}$ and $\hat{\mu}^{*}=\hat{\lambda}^{*}$.

Proof. (a) Given a solution $\hat{u}^{*}$ of (36) with associated multiplier $\hat{\lambda}^{*}$, one follows the lines before (38) in order to show (a).

(b) By optimality of $\hat{v}^{*}$ we have $\hat{M} \hat{v}^{*}-\hat{q}-\hat{\mu}^{*}=0$. Multiplying this identity by $\hat{M}^{-1}$ yields

$$
\hat{v}^{*}-\hat{\Lambda} \hat{L}^{-1} \hat{f}-\hat{\Lambda} \hat{L}^{-1} \hat{\Lambda}^{\top} \hat{\mu}^{*}=0 .
$$

Now we set $\hat{u}^{+}:=\hat{L}^{-1}\left(\hat{f}+\hat{\Lambda}^{\top} \hat{\mu}^{*}\right)$. From (40) and (38) we obtain

$$
\begin{aligned}
\hat{L} \hat{u}^{+}-\hat{f}-\hat{\Lambda}^{\top} \hat{\mu}^{*} & =0, \\
\hat{v}^{*}=\hat{\Lambda} \hat{u}^{+} & \geq 0, \\
\hat{\mu}^{*} & \geq 0 .
\end{aligned}
$$

From the uniqueness of the solution to (35) we conclude $\hat{u}^{+}=\hat{u}^{*}$ and $\hat{\mu}^{*}=$ $\hat{\lambda}^{*}$.

Combining Propositions 3.1 and 3.3 proves that $\left(P_{N}\right)$ and $(39)$ are equivalent in the sense that given the solution to one of the problems, then we can construct the solution to the other one.

Similarly to (35) we can now derive a semismooth Newton method of the type of Algorithm 2 for iteratively solving (38). For this purpose we introduce $\hat{\mu} \geq 0$ in (38) which then can equivalently be expressed as: Find $\hat{v}, \hat{\mu} \in \mathbb{R}^{|B|}$ such that

$$
\begin{aligned}
\hat{M} \hat{v}-\hat{q}-\hat{\mu} & =0, \\
\max (\alpha \hat{\mu}-\hat{v}, 0)-\alpha \hat{\mu} & =0 .
\end{aligned}
$$


Using the techniques in the proof of Proposition 3.3 one shows that if $\hat{v}^{(0)}=\hat{\Lambda} \hat{u}^{(0)}=\hat{\Lambda} u_{\tilde{B}}^{(0)}$ and $\hat{\mu}^{(0)}=\hat{\lambda}^{(0)}=\lambda^{(0)}$ then the iterates of the semismooth Newton methods for solving (41) and (35) coincide. Consequently, any convergence assertion associated to the semismooth Newton algorithm for (41) carries over to Algorithm 2 and vice versa.

3.3. Global convergence and monotonicity. Based on the results of the previous section, we now combine the local convergence results with global ones, i.e., convergence of Algorithm 2 regardless of the initialization.

Before we commence with the global convergence we emphasize the importance of (38), respectively (39). For this purpose let us for the moment assume that $\tilde{B}=B$ and $\hat{\Lambda}=E$ with $E$ denoting the $|B| \times|B|$-identity matrix, which corresponds to a unilateral constraint without jump. Further let $L$ be a M-matrix. Then the Schur complement $\hat{L}$ is a M-matrix too; see [2]. Observe that in the case of this example we have $\hat{M}=\hat{L}$. From $[6$, Theorem 3.1] it follows immediately that the semismooth Newton method converges globally and that the primal iterates tend monotonically to the solution.

Of course, in the case of the crack problem the M-matrix property as well as the assumption on $\hat{\Lambda}$ hold not true. However, in our numerical practice Algorithm 2 still behaves like a globally convergent method. In many cases we further observe monotonicity in the jump of the displacements across the crack along the iteration sequence. Moreover, as we confirmed numerically, frequently $\hat{M}$ is a small perturbation of a M-matrix. Based on these observations and relying on the reduced problem formulation (39), respectively (38), we adopt the global convergence and monotonicity results of $[6]$.

Let $\|\cdot\|_{1}$ denote the subordinate matrix norm corresponding to the vector 1-norm.

Theorem 3.2. Assume that $\hat{M}=\hat{K}+\hat{S}$ with $\hat{K} \in \mathbb{R}^{|B| \times|B|}$ a nonsingular M-matrix and with $\hat{S} \in \mathbb{R}^{|B| \times|B|}$ a perturbation such that $\|\hat{S}\|_{1}$ is sufficiently small. Then Algorithm 2 is well-defined, and $\left(\left(u^{(n)}, \lambda^{(n)}\right)\right)$ converges to $\left(u^{*}, \lambda^{*}\right)$, the solution of $(23)$, regardless of the initial choice. The local convergence rate is superlinear.

Proof. Apply [6, Theorem 3.2] to the semismooth Newton method associated to (41). Then use the equivalence of this algorithm to Algorithm 2.

Concerning the monotonous convergence of $\Lambda u^{(n)}$ we have the following two assertions. For their respective formulation we assume that $\hat{M}=\hat{K}+\hat{S}$, 
and we introduce the matrices

$$
\begin{aligned}
\hat{T}_{I I} & =\sum_{l=1}^{\infty}\left(-\hat{K}_{I I}^{-1} \hat{S}_{I I}\right)^{l}, \\
\hat{U}_{I A} & =\hat{T}_{I I} \hat{K}_{I I}^{-1} \hat{K}_{I A}+\hat{K}_{I I}^{-1} \hat{S}_{I A}+\hat{T}_{I I} \hat{K}_{I I}^{-1} \hat{S}_{I A}, \\
\hat{V}_{I I} & =\hat{T}_{I I} \hat{K}_{I I}^{-1},
\end{aligned}
$$

where $I, A$ are disjoint subsets of $B$. Note that these matrices are 0 when $S=0$.

The first monotonicity result is given be the next theorem.

Theorem 3.3. Let the assumptions of Theorem 3.2 be satisfied. For all $n \in \mathbb{N}_{0}$ and for all sets $\hat{A}^{(n)}=\left\{i \in B: \alpha \hat{\mu}_{i}^{(n)}-\hat{v}_{i}^{(n)}>0\right\}$ and $\hat{I}^{(n)}=\{i \in$ $\left.B: \alpha \hat{\mu}_{i}^{(n)}-\hat{v}_{i}^{(n)} \leq 0\right\}$ suppose that

$$
\left(\hat{K}_{\hat{I}^{(n)} \hat{I}^{(n)}}^{-1}+\hat{V}_{\hat{I}^{(n)} \hat{I}^{(n)}}\right) \geq 0
$$

and there exists an index $n_{0} \in \mathbb{N}_{0}$ with

$$
\begin{aligned}
& \text { either } \quad\left(\hat{K}_{\hat{I}^{\left(n_{0}\right)} \hat{I}^{\left(n_{0}\right)}}^{-1} \hat{K}_{\hat{I}^{\left(n_{0}\right)} \hat{A}^{\left(n_{0}\right)}}+\hat{U}_{\hat{I}^{\left(n_{0}\right)} \hat{A}^{\left(n_{0}\right)}}\right) \leq 0, \\
& \text { or } \quad \hat{v}^{\left(n_{0}\right)} \geq 0 .
\end{aligned}
$$

Then $\Lambda u^{*} \geq \Lambda u^{(n+1)} \geq \Lambda u^{(n)} \geq 0$ for all $n \geq n_{0}$.

Proof. Apply [6, Theorem 3.3] to the semismooth Newton method associated to (41), which yields $\hat{v}^{*} \geq \hat{v}^{(n+1)} \geq \hat{v}^{(n)} \geq 0$ for all $n \geq n_{0}$. Then use the facts that $\hat{v}^{(n)}=\hat{\Lambda} \hat{u}^{(n)}$ for all $n \in \mathbb{N}_{0}$ and $\Lambda=(\mathbf{0}, \hat{\Lambda})$.

Finally we state another monotonicity result which is based on a particular initialization of Algorithm 2.

Theorem 3.4. Let the assumptions of Theorem 3.2 be satisfied, and let the sets $\hat{A}^{(0)}, \hat{I}^{(0)}$ be defined as in Theorem 3.3. Suppose that Algorithm 2 is initialized with $\lambda^{(0)}=0$ and $u^{(0)}=\left(u_{D}^{(0)}, \hat{u}^{(0)}\right)$ with $\hat{u}^{(0)}=\hat{L}^{-1} \hat{f}$ and $u_{D}^{(0)}=L_{D D}^{-1}\left(f_{D}-L_{D} \tilde{B}^{(0)}\right)$. If for $\hat{v}^{(0)}=\hat{\Lambda} \hat{u}^{(0)}$ there holds

$$
\hat{v}_{\hat{I}^{(0)}}^{(0)}+\left(\hat{K}_{\hat{I}^{(0)} \hat{I}^{(0)}}^{-1} \hat{K}_{\hat{I}^{(0)} \hat{A}^{(0)}}+\hat{U}_{\hat{I}^{(0)} \hat{A}^{(0)}}\right) \hat{v}_{\hat{A}^{(0)}}^{(0)} \geq 0,
$$

and (42) is satisfied, then the sequence $\left(\Lambda u^{(n)}\right)$ converges monotonically and $\Lambda u^{(n)} \geq 0$ for all $n \geq 1$.

Proof. Observe that the assumption on $\lambda^{(0)}$ yields $\hat{\mu}^{(0)}=0$. Apply $[6$, Theorem 3.3] to the semismooth Newton method associated to (41), which yields the monotone convergence of $\left(\hat{v}^{(n)}\right)$ and $\hat{v}^{(n)} \geq 0$ for $n \geq 1$. Since $\hat{v}^{(n)}=\hat{\Lambda} \hat{u}^{(n)}, u^{(n)}=\left(u_{D}^{(n)}, \hat{u}^{(n)}\right)$, with $u_{D}^{(n)}=L_{D D}^{-1}\left(f_{D}-L_{D} \hat{B}^{(n)}\right)$, and $\Lambda=(\mathbf{0}, \hat{\Lambda})$ the assertion follows. 


\section{Numerical RESUlts}

Our numerical test problem of the type $(P)$ is given by the Lamé model on the unit square in $\mathbb{R}^{2}$ with three collinear cracks. For this configuration, we report on the results obtain from Algorithm 2 and compare these with the output obtained from a discretized version of Algorithm 1. Combined the results of Sections 2 and 3 indicate superlinear, but mesh-dependent, convergence of semismooth Newton methods applied to $(P)$. This will be confirmed by the numerical results. The number of iterations, however, only increases moderately as the mesh-size is decreased. Moreover, a continuation procedure with respect to the mesh-size will be introduced which significantly reduces the iteration number at finer mesh-sizes.

4.1. 2D-Lamé problem with three collinear cracks. For $d=2$ we choose $\Omega$ as the unit square

$$
\Omega=\left\{x=\left(x_{1}, x_{2}\right)^{\top} \in \mathbb{R}^{2}: \quad 0<x_{1}<1,\left|x_{2}\right|<0.5\right\}
$$

with the boundary $\partial \Omega$ consisting of the parts:

$$
\begin{aligned}
& \Gamma_{D}=\left\{x: x_{1}=1,\left|x_{2}\right| \leq 0.5\right\}, \quad \Gamma_{N}=\Gamma_{N 1} \cup \Gamma_{N 2}^{ \pm}, \\
& \Gamma_{N 1}=\left\{x: x_{1}=0,\left|x_{2}\right| \leq 0.5\right\}, \quad \Gamma_{N 2}^{ \pm}=\left\{x: 0<x_{1}<1, x_{2}= \pm 0.5\right\} .
\end{aligned}
$$

Three collinear cracks in $\Omega$ are given by

$$
\Gamma_{C}=\cup_{n=1}^{3} \Gamma_{C n}, \quad \text { with } \Gamma_{C n}=\left\{x: 0<x_{1}<0.9, x_{2}=0.5-0.25 n\right\}
$$

as depicted in Figure 2.

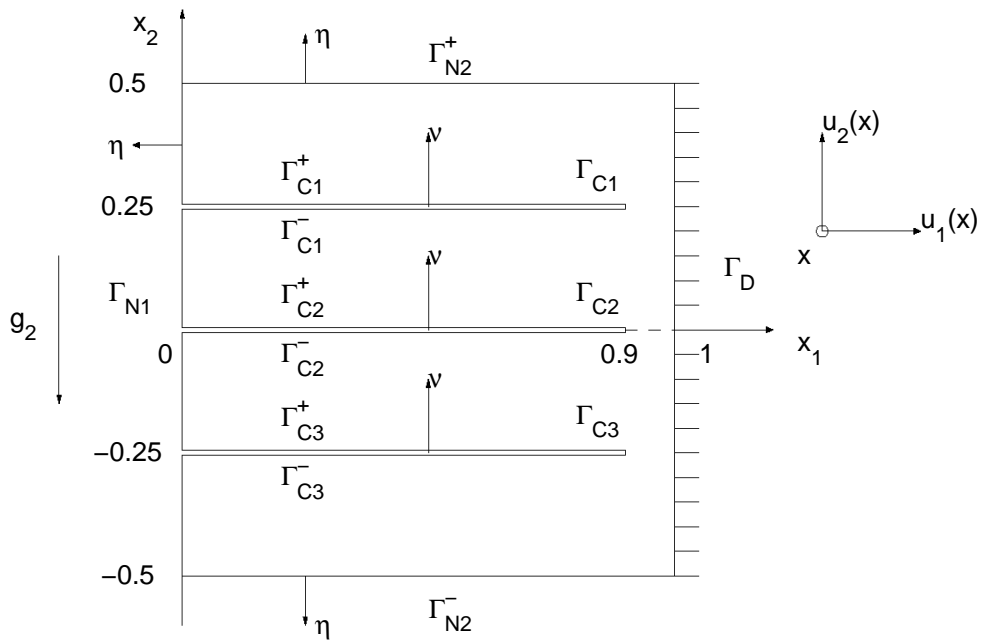

Figure 2. Domain $\Omega_{C}$ with three cracks $\Gamma_{C 1}, \Gamma_{C 2}, \Gamma_{C 3}$. 
The unit normal vector on $\Gamma_{C}$ is $\nu=(0,1)^{\top}$. Whence the non-penetration condition becomes

$$
\llbracket u_{2} \rrbracket(x) \geq 0 \quad \text { for all } x \in \Gamma_{C} .
$$

The plane-stress Lamé model of an isotropic solid is given in terms of the stress tensor

$$
\begin{aligned}
& \sigma_{11}(u)=\left(2 \mu_{L}+\lambda_{L}\right) \varepsilon_{11}(u)+\lambda_{L} \varepsilon_{22}(u), \quad \sigma_{12}(u)=2 \mu_{L} \varepsilon_{12}(u), \\
& \sigma_{22}(u)=\lambda_{L} \varepsilon_{11}(u)+\left(2 \mu_{L}+\lambda_{L}\right) \varepsilon_{22}(u), \quad \mu_{L}=\frac{E}{2\left(1+\nu_{L}\right)}, \quad \lambda_{L}=\frac{2 \nu_{L} \mu_{L}}{1-2 \nu_{L}},
\end{aligned}
$$

where the strain tensor is given by

$$
\varepsilon_{11}(u)=u_{1,1}, \quad \varepsilon_{22}(u)=u_{2,2}, \quad \varepsilon_{12}(u)=0.5\left(u_{1,2}+u_{2,1}\right) .
$$

We choose the material parameters $0<\nu_{L}<0.5$ and $E=7.3 \times 10^{4}(\mathrm{mPa})$.

The solid is assumed to be clamped at $\Gamma_{D}$, stress-free at $\Gamma_{N 2}^{ \pm}$, and loaded at $\Gamma_{N 1}$ with traction $g=\left(g_{1}, g_{2}\right)^{\top}$ as illustrated in Figure 2. We choose $g_{1}=0$ and $g_{2}=-10^{-3} \mu_{L}$. This results in the following relations:

$$
\begin{gathered}
-\sigma_{11,1}(u)-\sigma_{12,2}(u)=-\sigma_{12,1}(u)-\sigma_{22,2}(u)=0 \text { in } \Omega_{C}, \\
u_{1}=u_{2}=0 \quad \text { on } \Gamma_{D}, \quad \pm \sigma_{12}(u)= \pm \sigma_{22}(u)=0 \quad \text { on } \Gamma_{N 2}^{ \pm}, \\
-\sigma_{11}(u)=0, \quad-\sigma_{12}(u)=g_{2} \quad \text { on } \Gamma_{N 1} .
\end{gathered}
$$

For the discretization of our test problem $(P)$ we use standard linear finite elements and construct a uniform triangulation. By $h>0$ we denote the corresponding mesh size.

We apply Algorithm 2 with fixed $\alpha \in\left(10^{-4}, 10^{-3}\right)$. The initial choice is given by $y^{0}=\left(u^{0}, \lambda^{0}\right)^{\top}=\left(L^{-1} f, 0\right)^{\top}$.

In Figure 3 we depict the domain $\Omega_{C}$ in Lagrange coordinates $x+u^{*}(x)$ for two different mesh sizes $h$. One can clearly observe the effect of the surface traction applied at $x_{1}=0$.
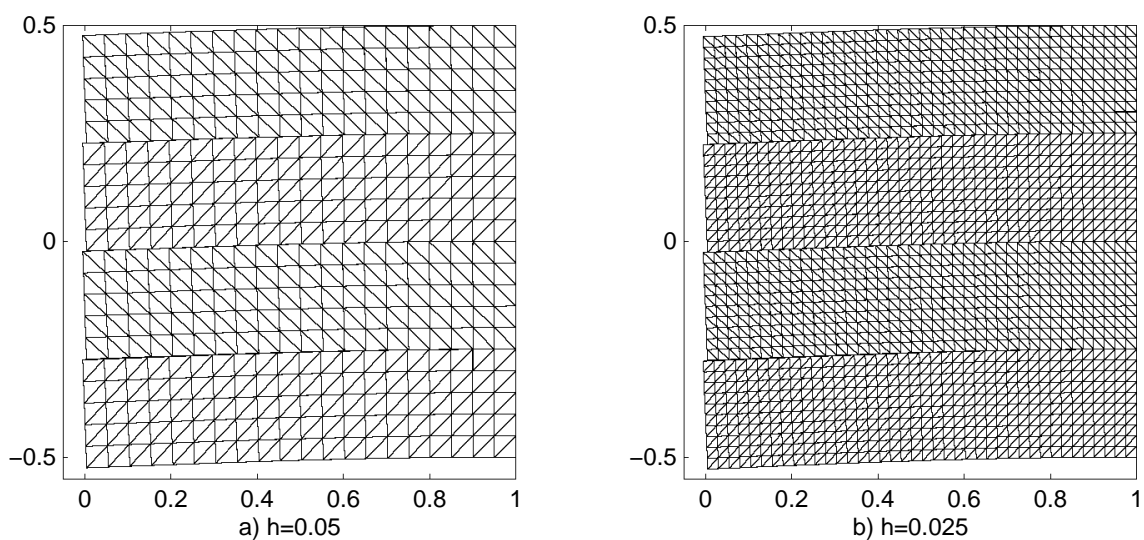

Figure 3. Domain $\Omega_{C}$ in Lagrange coordinates $x+u^{*}(x)$. 
From our numerical tests we can report on the following behavior of Algorithm 2:

- In all cases tested the algorithm terminated after finitely many iterations by producing the same active/inactive set structure in two consecutive iterations and, thus, it found the exact solution of the discretized problem $\left(P_{N}\right)$.

- In the example presented below it terminated successfully in 5 iterations for the mesh size $h=0.05,7$ for $h=0.025,7$ for $h=0.0125$, and 9 for $h=0.00625$. The number of iterations until successful termination increases only moderately as $h$ decreases.

In the sequel let us fix $h=0.025$ if not otherwise stated.

Next we discuss the influence of the parameter $\kappa_{L}=\frac{\lambda_{L}+\mu_{L}}{\mu_{L}}=\frac{1}{1-2 \nu_{L}}>0$. Note that the Lamé problem degenerates in the case where $\kappa_{L}=0$, i.e., there is no solution available. In addition to the behavior of Algorithm 2 described above, we observe frequently monotonous convergence of the iterates $\Lambda u^{(n)}$ describing the jump as depicted in Figure 4, i.e.,

$$
0 \leq \Lambda u^{(1)} \leq \Lambda u^{(2)} \leq \ldots .
$$

This confirms our results on monotonous global convergence, Theorem 3.3
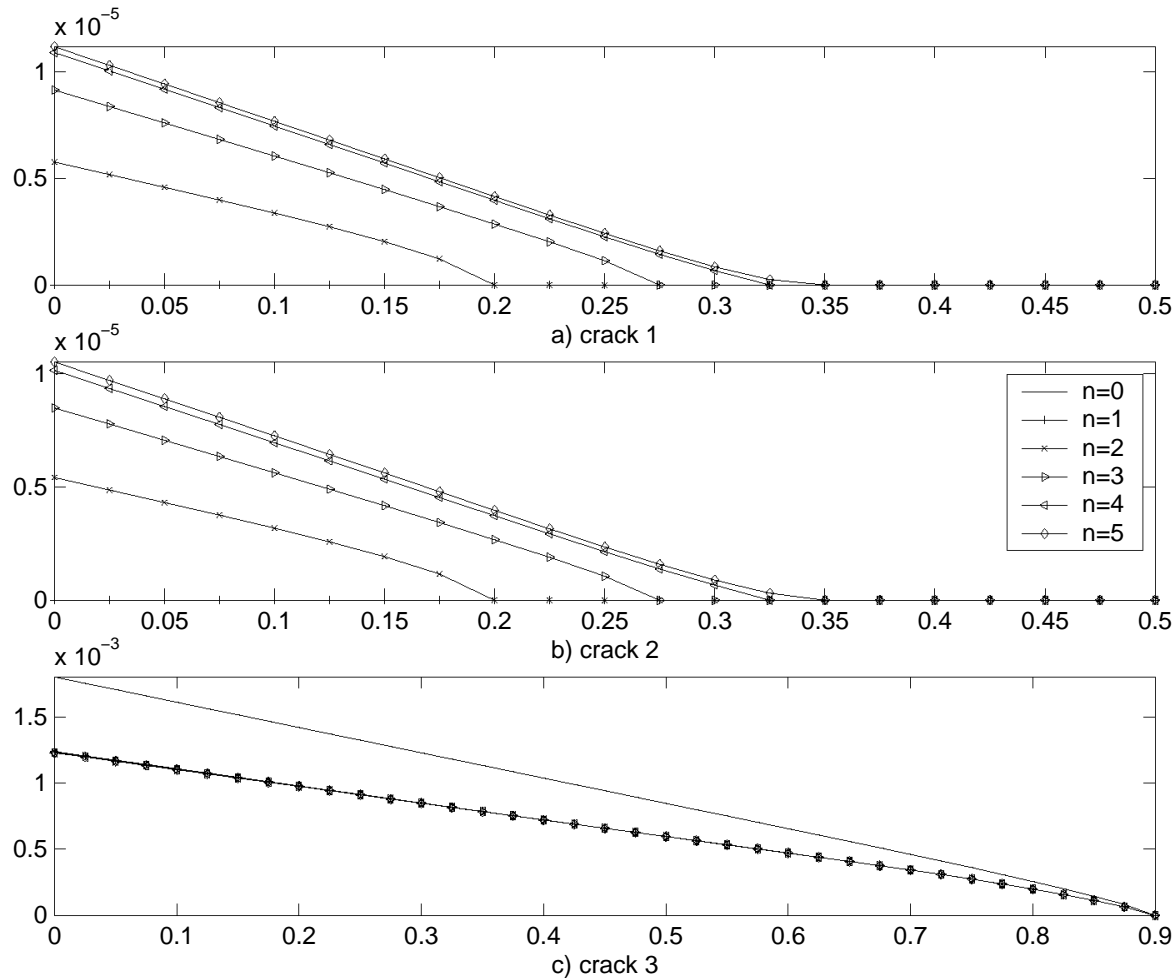

FiguRE 4. Iterates $\Lambda u^{(n)}$ of the jump for $\nu_{L}=0.34$. 
and Theorem 3.4. In our tests this monotonous behavior was destroyed when $\nu_{L} \approx 0$, i.e., $\lambda_{L} \approx 0$ and $\kappa_{L} \approx 1$. The number of iterations, however, appeared to be stable w.r.t. small $\nu_{L}$ as can be seen from Figures 5 and 6 which document the jumps $\Lambda u^{(n)}$ across the crack and the corresponding Lagrange multipliers $\lambda^{(n)}$ for $\nu_{L}=0.0001$. Note that $\Lambda u^{(n)}$ for crack 3 is always positive and, thus, implies $\lambda^{(n)}=0$ for all $n$. We point out that
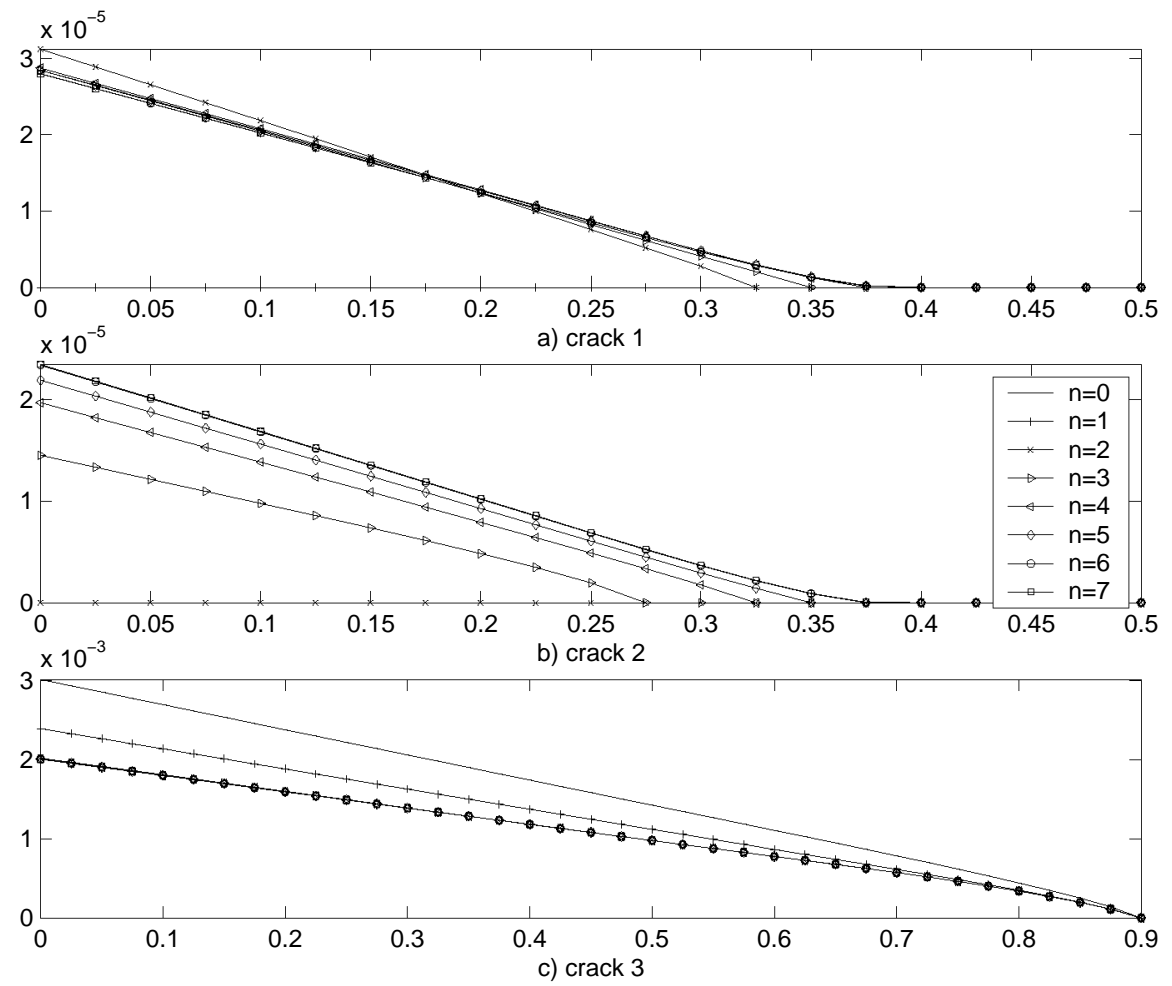

FiguRE 5. Iterates $\Lambda u^{(n)}$ of the jump for $\nu_{L}=0.0001$.

we depict only the intervals with $x_{1} \in(0,0.5)$ for the 1st and 2nd crack, respectively, since $\left(\Lambda u^{(n)}\right)_{i}=0$ at nodal points with $x_{1} \in(0.5,0.9)$. From Figure 5 it immediately follows that all iterates are feasible, i.e.,

$$
\Lambda u^{(n)} \geq 0 \text { for all } n \geq 1 \text {. }
$$

Finally, we report on the behavior of Algorithm 2 when using a continuation technique for its initialization. This method works as follows: We solve problem $\left(P_{N}\right)$ on a grid with a coarse mesh size $H>0$ and use the prolongation of this solution and its corresponding multipliers onto the finer mesh with mesh size $h<H$ as initial values. In Table 1 the first row shows the number of iterations required by Algorithm 2 when initialized by $u^{(0)}=L^{-1} f$ and $\lambda^{(0)}=0$ for the specified mesh size (fixed grid method). The second row presents the results when only the coarse problem with 

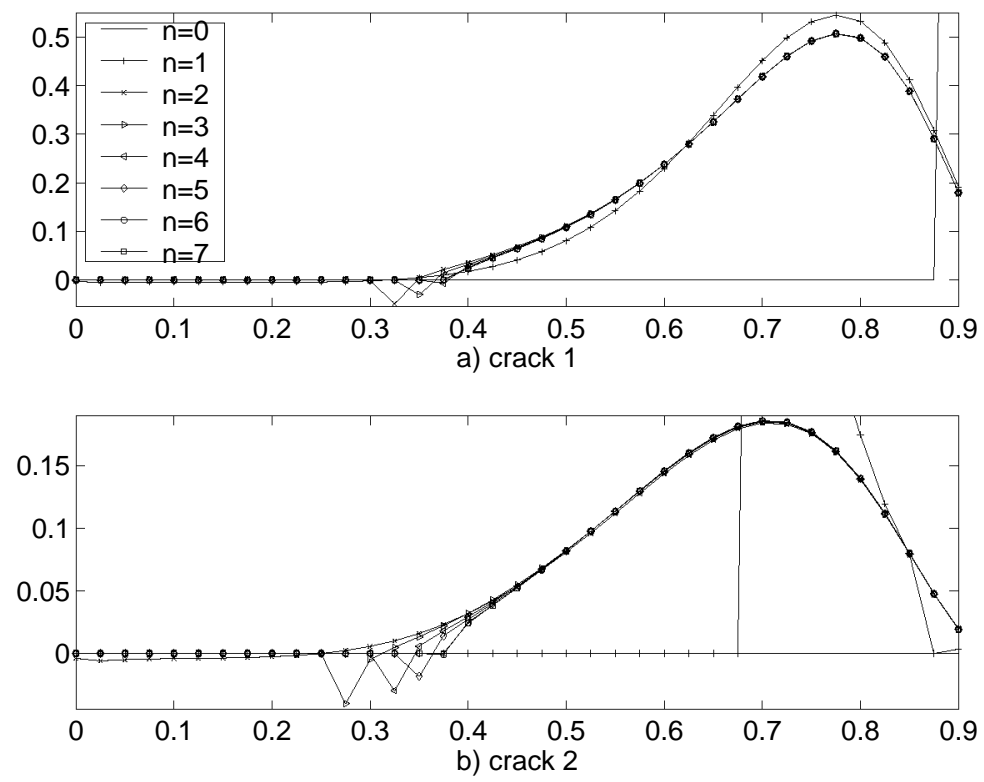

Figure 6. Iterates $\lambda^{(n)}$ of the multiplier.

$h=0.05$ is initialized as described above and in all other cases the continuation technique is used. The entry ' +2 ' indicates that based on the special initialization subsequently only two iterations are required on the next finer grid for successful termination. By this the second iteration confirmed in step 2 the same active/inactive sets obtained from the first iteration. The results indicate that the continuation technique is an effective tool for reducing costly fine grid iterations.

\begin{tabular}{|c|c|c|c|c|}
\hline \#it & $h=0.05$ & $h=0.025$ & $h=0.0125$ & $h=0.00625$ \\
\hline fixed grid & 5 & 7 & 7 & 9 \\
\hline continuation & 5 & +2 & +2 & +2 \\
\hline
\end{tabular}

TABLE 1. \# of iteration for fixed grid and the continuation method.

4.2. Discrete penalization approach. For the discretization of Algorithm 1 applied to the Lamé problem with the three collinear cracks we use the same technique as described in the previous section.

For every fixed $\gamma>0$ Algorithm 1 terminated successfully at the solution to the discretized version of problem $\left(P_{\gamma}\right)$ with an iteration count similar to Algorithm 2.

In Figures 7 and 8 we depict the iterates $\Lambda u^{(n)}$ of the jump and the discrete active sets, respectively, for $\gamma=10^{5}$. From Figure 7 it is apparent that the iterates $\Lambda u^{(n)}$ are not monotonous, and that they are infeasible for 

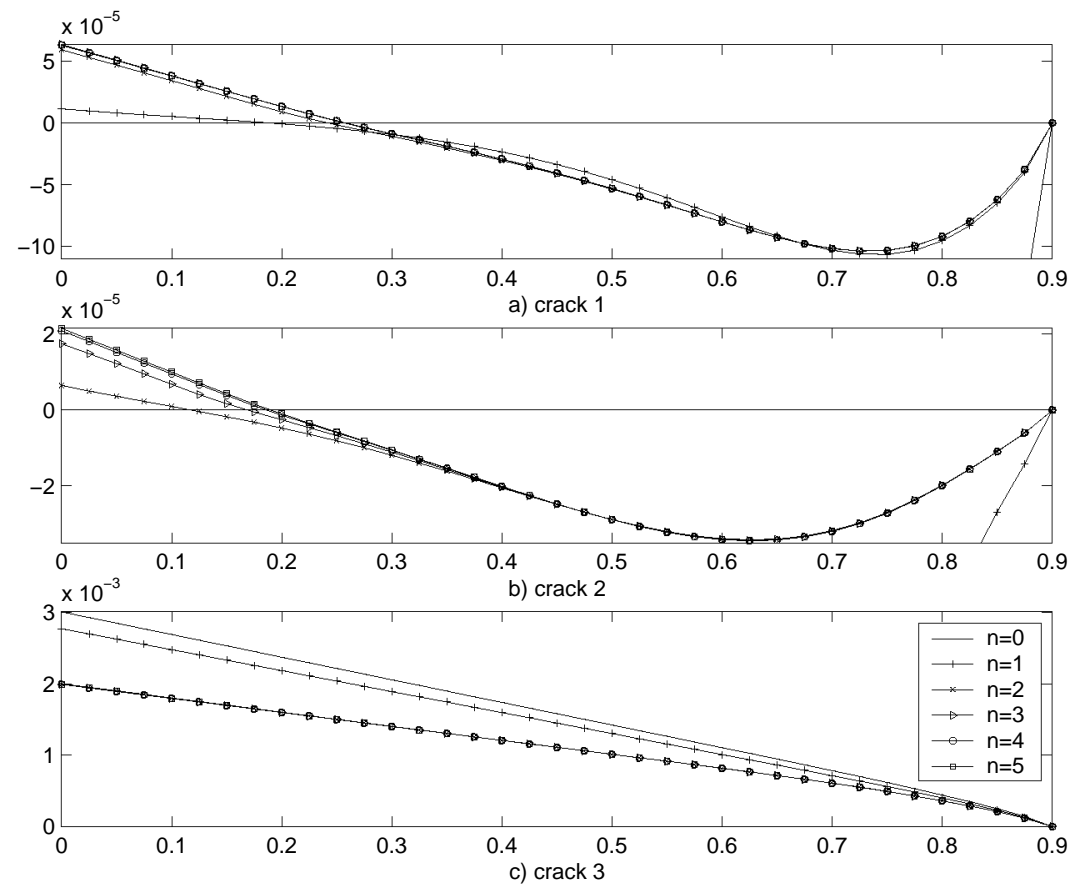

FiguRE 7. Iterates $\Lambda u^{(n)}$ of the jump for $\log _{10} \gamma=5$.
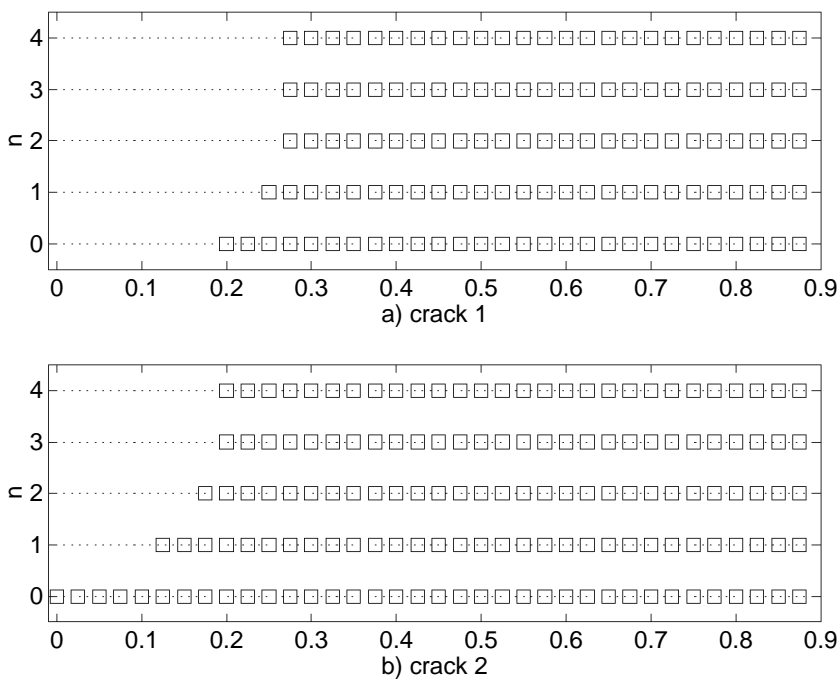

FiguRE 8. History of the active set as $\log _{10} \gamma=5$.

cracks 1 and 2. Figure 8, however, shows clearly that the active/inactive sets converge monotonically. The same behavior was observed for all values $\log _{10} \gamma=1, \ldots, 9$ tested. 
Next we study the approximation quality as $\gamma \rightarrow+\infty$. We recall that Proposition 2.1 asserts that $u^{\gamma} \rightarrow u^{*}$ as $\gamma \rightarrow+\infty$ in the continuous setting. In Figure 9 we show the relative error between the discrete analogues $u_{h}^{\gamma}$ and $u_{h}^{*}$ of $u^{\gamma}$ and $u^{*}$, respectively, with respect to the relative discrete $H^{1}$-norm given by

$$
q\left(\gamma^{-1}\right)=\left(\frac{\left(u_{h}^{\gamma}-u_{h}^{*}\right)^{\top} L\left(u_{h}^{\gamma}-u_{h}^{*}\right)}{\left(u_{h}^{*}\right)^{\top} L u_{h}^{*}}\right)^{1 / 2} .
$$

Here $u_{h}^{*}$ denotes the solution obtained by Algorithm 2. For the various meshsizes $h$ we use a logarithmic scale on the horizontal axis. As we can see, $q\left(\gamma^{-1}\right)$ depends only weakly on $h$. Further $u_{h}^{\gamma}$ is a very close approximation of $u_{h}^{*}$ for large $\gamma$. In fact, for $\log _{10} \gamma \geq 7$ both solutions are almost identical, difference in the sup-norm is less then 0.00035 .

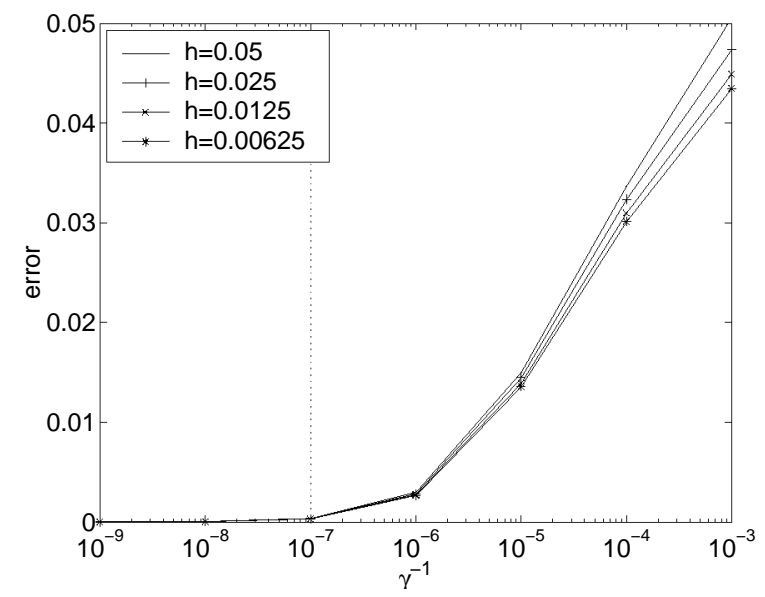

FiguRE 9. The relative error $q\left(\gamma^{-1}\right)$.

Figure 10 studies the influence of $\gamma$ on the active set at the numerical solution $u_{h}^{\gamma}$ compared to the active set of the true discrete solution $u_{h}^{*}$. We observe that for $\log _{10} \gamma \geq 7$ the active sets at the optimal solutions $u_{h}^{\gamma}$ and $u_{h}^{*}$ coincide. This is another instance of the fact that $u_{h}^{\gamma}$ closely approximates $u_{h}^{*}$ for large $\gamma$.

Finally we report on the comparison of Algorithm 1 and Algorithm 2 w.r.t. the number of iterations until successful termination. The results are displayed in Table 2. For fixed $\gamma$ we observe that the number of iterations stabilizes as $h$ is reduced. This reflects our theoretical findings in section 2 . On the other hand, for sufficiently small but fixed mesh size $h$ the number of iterations required by Algorithm 1 is increasing as $\gamma$ increases. This comes from the fact that the superlinear rate proved in section 2 depends on $\gamma$. Indeed, from Theorem 2.1 we infer that the radius of the ball about $u_{h}^{\gamma}$ where superlinear convergence is guaranteed tends to zero as $\gamma \rightarrow \infty$. 

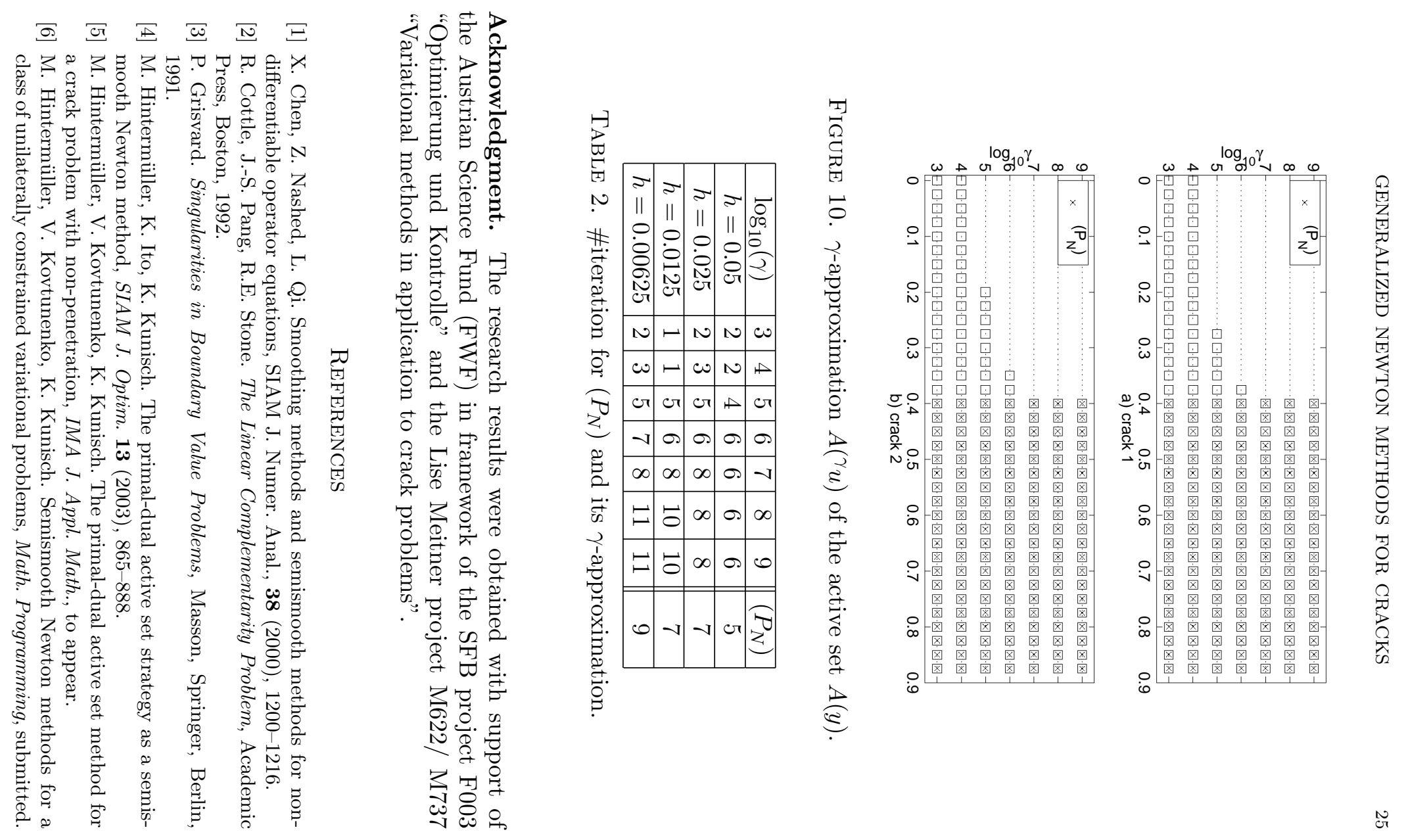
[7] G.C. Hsiao, E. Schnack, W.L. Wendland. Hybrid methods for boundary value problems via boundary energy, in The Maz'ya Anniv. Collection, Vol.2, (Rostock, 1998), 165-184, Birkhäuser, Basel, 1999.

[8] K. Ito, K. Kunisch. Augmented Lagrangian methods for nonsmooth convex optimization in Hilbert spaces, Nonlinear Anal. Theory Meth. Appl. 41 (2000), 573-589.

[9] K. Ito, K. Kunisch. Semi-smooth Newton methods for the variational inequalities of the first kind, ESAIM, Math. Modelling Numer. Anal., 37 (2003), 41-62.

[10] A.M. Khludnev, V.A. Kovtunenko. Analysis of Cracks in Solids, WIT-Press, Southampton, Boston, 2000.

[11] A.M. Khludnev, J. Sokolowski. The Griffith formula and the Cherepanov-Rice integral for crack problems with unilateral conditions in nonsmooth domains, Euro. J. Appl. Math. 10 (1999), 379-394.

[12] D. Klatte, B. Kummer. Nonsmooth Equations in Optimization, Kluwer Publishers, Dordrecht, 2002.

[13] V. Kovtunenko. Numerical simulation of the nonlinear crack problem with nonpenetration, Math. Meth. Appl. Sci., to appear.

[14] G. Kuhn. Boundary element technique in elastostatics and linear fracture mechanics. Theory and engineering applications, in CISM Courses Lect., 301, 109-169, Springer, Vienna, 1988.

[15] W.G. Mazja, S.A. Nazarow, B.A. Plamenewski. Asymptotische Theorie elliptischer Randwertaufgaben in singulär gestörten Gebieten, Akademie-Verlag, Berlin, 1991.

[16] R. Mifflin. Semismooth and semiconvex functions in constrained optimization, SIAM J. Control Optim. 15 (1977), 959-972.

[17] V.V. Prasolov. Problems and Theorems in Linear Algebra. AMS, Providence, 1994.

[18] L. Qi, J. Sun. A nonsmooth version of Newton's method, Math. Prog. 58 (1993), 353-367.

[19] M. Ulbrich. Semismooth Newton methods for operator equations in function spaces, SIAM J. Optim., 13 (2003), 805-842. 